\title{
A new take on the low-mass brown dwarf companions on wide orbits in Upper-Scorpius ${ }^{\star}$
}

\author{
S. Petrus ${ }^{1}$, M. Bonnefoy ${ }^{1}$, G. Chauvin ${ }^{2,1}$, C. Babusiaux ${ }^{1}$, P. Delorme ${ }^{1}$, A.-M. Lagrange ${ }^{1}$, N. Florent ${ }^{1}$, A. Bayo ${ }^{3,4}$, \\ M. Janson ${ }^{5}$, B. Biller ${ }^{6}$, E. Manjavacas ${ }^{7}$, G.-D. Marleau ${ }^{8}$, and T. Kopytova ${ }^{9,10}$ \\ ${ }^{1}$ Univ. Grenoble Alpes, CNRS, IPAG, 38000 Grenoble, France \\ e-mail: simon.petrus@univ-grenoble-alpes.fr \\ ${ }^{2}$ Unidad Mixta Internacional Franco-Chilena de Astronomía, CNRS/INSU UMI 3386 and Departamento de Astronomía, \\ Universidad de Chile, Casilla 36-D, Santiago, Chile \\ ${ }^{3}$ Instituto de Física y Astronomía, Universidad de Valparaíso, Valparaíso, Chile \\ ${ }^{4}$ Núcleo Milenio de Formación Planetaria (NPF), Valparaiso, Chile \\ ${ }^{5}$ Department of Astronomy, Stockholm University, AlbaNova University Center, 10691 Stockholm, Sweden \\ ${ }^{6}$ Institute for Astronomy, The University of Edinburgh Royal Observatory, Blackford Hill, Edinburgh EH9 3HJ, UK \\ ${ }^{7}$ W. M. Keck Observatory 65-1120 Mamalahoa Hwy. Kamuela, HI 96743, USA \\ ${ }^{8}$ Institut für Astronomie und Astrophysik, Eberhard Karls Universität Tübingen, Auf der Morgenstelle 10, \\ 72076 Tübingen, Germany \\ ${ }^{9}$ School of Earth \& Space Exploration, Arizona State University, Tempe AZ 85287, USA \\ ${ }^{10}$ Ural Federal University, Yekaterinburg 620002, Russia
}

Received 18 April 2019/ Accepted 27 September 2019

\begin{abstract}
Context. The Upper-Scorpius association (5-11 Myr) contains a unique population of low-mass $\left(M \leq 30 M_{\text {Jup }}\right)$ brown dwarfs either free-floating, forming wide pairs, or on wide orbits around solar-type and massive stars. The detailed relative characterization of their physical properties (mass, radius, temperature, composition, and ongoing accretion) offers the opportunity to potentially explore their origin and mechanisms of formation.

Aims. In this study, we aim to characterize the chemical and physical properties of three young, late-M brown dwarfs claimed to be companions of the Upper-Scorpius stars USco 161031.9-16191305, HIP 77900, and HIP 78530 using medium-resolution spectroscopy at UV (0.30-0.56 $\left.\mu \mathrm{m} ; R_{\lambda} \sim 3300\right)$, optical ( $\left.0.55-1.02 \mu \mathrm{m} ; R_{\lambda} \sim 5400\right)$, and NIR (1.02-2.48 $\left.\mu \mathrm{m} ; R_{\lambda} \sim 4300\right)$ wavelengths. The spectra of six free-floating analogs from the same association are analyzed for comparison and to explore the potential physical differences between these substellar objects found in different configurations. We also aim to examine and analyze hydrogen emission lines at UV and optical wavelengths to investigate the presence of ongoing accretion processes.

Methods. The X-shooter spectrograph at VLT was used to obtain the spectra of the nine young brown dwarfs over the $0.3-2.5 \mu \mathrm{m}$ range simultaneously. Performing a forward modeling of the observed spectra with the ForMoSA code, we infer the $T_{\text {eff }}, \log (g)$, and radius of our objects. The code compares here the BT-SETTL15 models to the observed spectra using the Nested Sampling Bayesian inference method. Mass is determined using evolutionary models, and a new analysis of the physical association is presented based on Gaia-DR2 astrometry.

Results. The $T_{\text {eff }}$ and $\log (g)$ determined for our companions are compatible with those found for free-floating analogs of the Upper-Scorpius association and with evolutionary model predictions at the age of the association. However the final accuracy on the $T_{\text {eff }}$ estimates is strongly limited by nonreproducibility of the BT-SETTL15 models in the range of $T_{\text {eff }}$ corresponding to the M8-M9 spectral types. We identified $\mathrm{H}_{\alpha}, \mathrm{H}_{\beta}, \mathrm{H}_{\gamma}$, and $\mathrm{Ca}$ II H and $\mathrm{K}$ emission lines in the spectrum of several objects. We attribute these lines to chromospheric activity except for the free-floating object USco 1608-2315 for which they are indicative of active accretion $\left(\dot{M} \leq 10^{-10.76} M_{\odot} \mathrm{yr}^{-1}\right)$. We confirm the four-fold over-luminosity of USco 161031.9-16191305 B down to $0.3 \mu \mathrm{m}$, which could be explained in part by the activity of this object and if the companion is an unresolved multiple system.
\end{abstract}

Key words. brown dwarfs - stars: atmospheres - stars: fundamental parameters - stars: luminosity function, mass function planetary systems

\section{Introduction}

The first brown dwarfs (BDs) were contemporaneously discovered with the first exoplanets at the end of the last millennium (Mayor \& Queloz 1995; Nakajima et al. 1995; Rebolo et al. 1995). Since then, thousands of BDs have been detected, and

\footnotetext{
${ }^{\star}$ Based on observations collected at the European Organisation for Astronomical Research in the Southern Hemisphere under ESO program 093.C-0769.
}

studied in isolation (Cushing et al. 2005; Kirkpatrick 2005) in the field or as wide- or short-period companions to nearby stars (Sahlmann et al. 2011; Allers 2012). Bridging the gap between planets and stars, BDs are too light by definition to burn hydrogen but are massive enough to burn deuterium (Burrows et al. 1997). Despite two decades of intensive study of BDs, many fundamental questions remain unanswered regarding their formation and evolution processes, their physical and atmosphere properties, and their connection to stars and planets. Multiple stellar-like formation pathways have been proposed for 
these objects: (i) turbulent fragmentation of molecular clouds (Padoan \& Nordlund 2004), (ii) premature ejection of protostellar embryos (Bate et al. 2002), (iii) photo-erosion of prestellar cores (Hester et al. 1996), and (iv) disk instability (e.g., Stamatellos \& Whitworth 2009). Studies of young star-forming regions are currently ongoing to identify differences in binary statistics linked to these different processes (e.g., Marks et al. 2017; Thies et al. 2015). Alternatively, we know from observations and theory that planetary formation mechanisms like core accretion are probably forming very massive giant planets, and populating the mass distribution of substellar companions up to $M \leq 35 M_{\text {Jup }}$ (e.g., Mordasini et al. 2009, 2012). There is therefore little doubt that both stellar and planetary formation mechanisms overlap in mass distribution, and an interesting question is to investigate whether stellar and planetary mechanisms might lead to different atmospheric properties that could be traced through observations. Core accretion might indeed lead to an overabundance of heavy elements in the atmosphere. Our ability to identify such chemical imprinting is very challenging, is beyond observational limitations, and is directly connected to our understanding and modeling of the physics of BD and exoplanet atmospheres. Confronting the latest predictions of substellar atmosphere models with high-quality optical and infrared spectra of young brown dwarfs is a key step toward this goal.

Evolutionary models predict how BDs contract and cool down with time, and how their fundamental parameters such as effective temperature, surface-gravity, radius, and luminosity evolve (Baraffe et al. 2003). The contraction leads to a decrease of the radius and an increase of the surface-gravity. This evolution impacts the bolometric luminosity and the spectrum with a modification of the pseudo-continuum and the appearance of atomic and molecular absorption lines at different evolutionary stages. Spectral morphology has been used for years to extend the old stellar spectral classification into the substellar one, from M-dwarfs to L-, T-, and Y-dwarfs reaching effective temperatures as cold as $T_{\text {eff }} \leq 450 \mathrm{~K}$ (Kirkpatrick et al. 2012; Kopytova et al. 2014). The fine characterization of the $\mathrm{BD}$ atmospheres therefore improves our global understanding of their physical properties, and also of their formation and evolution.

There are currently two approaches for the spectral characterization of BDs. The first is empirical and based on comparison with libraries of known young and old BDs (e.g., Allers \& Liu 2013; Bonnefoy et al. 2014; Bayo et al. 2017), and is tightly connected and limited by the size and the diversity of these libraries. A complementary alternative is the comparison of the observed spectra to the recent models of substellar atmospheres (e.g., Allard et al. 2001; Helling \& Woitke 2006; Barman et al. 2011; Madhusudhan et al. 2011; Morley et al. 2012; Tremblin et al. 2017; Charnay et al. 2018) of BDs and giant planets. This offers the advantage that the physical parameters for a given model can be derived independently of other observations, and that the influence of new physical ingredients can be tested (e.g., new atomic and molecular line opacities, presence and properties of clouds, nonequilibrium chemistry, thermochemical instability, etc.). Nevertheless, this latter approach is hindered by our limited observational knowledge and constraints to disentangle the relative importance of the tested physical parameters, eventually leading to significant degeneracies and systematic errors when using different families of atmosphere models or simply different sets of physical parameters. Both approaches remain therefore highly complementary today.

The detection of forming companions is a new observational window on the initial conditions of planetary systems (formation zone, timescales, and modes; accretion physics; e.g., Mordasini et al. 2017). Thus far, $\mathrm{H}_{\alpha}(656.3 \mathrm{~nm})$ and $\mathrm{Pa}_{\beta}(1282.2 \mathrm{~nm})$ lines have been detected in the spectra of eight companions with masses below $30 M_{\text {Jup }}$ and ages in the 1-10 Myr range (TWA $5 \mathrm{~B}$, GQ Lup B, CT Cha B, USco CTIO 108 B, DH Tau B, GSC 06214-00210 B, SR 12 C, PDS 70 B, Neuhäuser et al. 2000; Seifahrt et al. 2007; Schmidt et al. 2008; Béjar et al. 2008; Bonnefoy et al. 2014; Bowler et al. 2011; Santamaría-Miranda et al. 2018; Wagner et al. 2018; Zhou et al. 2014; Wu et al. 2017b). These lines are known tracers of active accretion and of substellar chromospheric activity. Submillimeter observations of these objects have so far failed to reveal excess emission from the expected mass reservoir (circumplanetary disk) surrounding these objects (Dai et al. 2010; Bowler et al. 2015; MacGregor et al. 2017; Wolff et al. 2017; Wu et al. 2017a,b) and only one companion, GSC 06214-00210 B, shows clear excess emission at near-infrared (NIR) wavelengths (Bowler et al. 2011). This might indicate that these companions bear very compact and optically thick disks (Wu et al. 2017b).

The Upper-Scorpius subgroup (hereafter Upper-Sco) in the Scorpius-Centaurus OB association contains one of the nearest $(d=146 \pm 3$ pc; de Bruijne 1999; de Zeeuw et al. 1999; Galli et al. 2018) and richest populations of young stars and substellar objects (e.g. Ardila et al. 2000; Lodieu et al. 2007, 2018; Luhman et al. 2018) down to the planetary-mass range. The extinction is low in this region $\left(A_{\mathrm{V}} \leq 2 \mathrm{mag}\right.$; Walter et al. 1994; Lallement et al. 2019). At an estimated age of 5-11 Myr (Pecaut et al. 2012; David et al. 2019), stars harbor primordial, transitional, and debris disks (Luhman \& Mamajek 2012; Esplin et al. 2018) suggesting planet or BD formation within disks at different completion levels. Upper-Sco also contains a large sample of low-mass BDs $\left(M<30 M_{\text {Jup }}\right)$ and planetary mass companions identified with deep-imaging and high-contrast imaging techniques. These companions are found over a wide range of projected separations ( 300-3400 au; Béjar et al. 2008; Lafrenière et al. 2008, 2010, 2011; Ireland et al. 2011; Aller et al. 2013) from M 7- to B6-type stars. Some of the free-floating lowmass BDs and companions harbor disks (e.g., Bowler et al. 2011; Dawson et al. 2013) and are actively accreting (Herczeg et al. 2009; Bowler et al. 2011; Lodieu et al. 2018). This unique population of low-mass BDs and planetary mass objects with various configurations (as companions, binaries, free-floating) and likely diverse origins represents a unique test bed for planet and BD formation models.

In this paper, we present a new study of three young BD companions to the stars HIP 78530, HIP 77900, and USco 161031.916191305 (hereafter USco 1610-1913), members of the UpperScorpius association. We obtain VLT/X-shooter $0.3-2.5 \mu \mathrm{m}$ spectra of these objects, and use them to characterize their physical properties. The data notably extend previous analysis of these objects to the optical at medium resolving powers, thus enabling investigation of emission lines related to accretion, and testing of the atmospheric models of young BDs. We provide an up-todate description of our targets in Sect. 2. The observations and the data reduction are detailed in Sect. 3. In Sect. 4, we present our results using both empirical and synthetic model approaches. Our ForMoSA forward modeling tool is used to explore the different atmospheric models and determine the most probable physical properties of these three companions. In Sect. 5, we focus our study on the emission-line properties observed for the three companions. In Sect. 6, we finally summarize and discuss our results in the context of previous work, and present perspective of future studies. 
Table 1. Description of the properties of the three systems HIP 78530, HIP 77900, and USco 1610-1913.

\begin{tabular}{|c|c|c|c|c|c|c|c|c|c|}
\hline \multirow[b]{2}{*}{ Source } & \multirow[b]{2}{*}{$d^{(a)}(\mathrm{pc})$} & \multirow[b]{2}{*}{$A_{\mathrm{v}}{ }^{(b)}$ (mag) } & \multicolumn{2}{|l|}{ Primary } & \multicolumn{4}{|c|}{ Companion } & \multirow[b]{2}{*}{ Ref. } \\
\hline & & & $T_{\text {eff }}(\mathrm{K})$ & $\mathrm{SpT}$ & $T_{\text {eff }}(\mathrm{K})$ & SpT & $\operatorname{Mass}\left(M_{\text {Jup }}\right)$ & Separation (AU) & \\
\hline USco 1610-1913 B & $143.9 \pm 8.0$ & 0.13 & $4140 \pm 150$ & K7 & $2400 \pm 150$ & $\mathrm{M} 9 \pm 0.5$ & $20 \pm 5$ & $779 \pm 9$ & (c) \\
\hline HIP $77900 \mathrm{~B}$ & 150.8 & 0.07 & $13700 \pm 1500$ & $\mathrm{~B} 6 \pm 1$ & 2400 & M9= & $20 \pm 7$ & $3200 \pm 300$ & (c) \\
\hline HIP $78530 \mathrm{~B}$ & $137.2 \pm 1.5$ & 0.075 & $\simeq 10500$ & B9V & $2700 \pm 100$ & M $7 \pm 0.5$ & $23 \pm 2$ & $623 \pm 8$ & (d) \\
\hline
\end{tabular}

References. ${ }^{(a)}$ Galli et al. (2018); ${ }^{(b)}$ Lallement et al. (2019); ${ }^{(c)}$ Aller et al. (2013); ${ }^{(d)}$ Lachapelle et al. (2015).

\section{Target description}

The three companions have close physical properties and spectral types (M8-M9), yet they were selected because they come with different configurations (mass ratio with the host star, projected separation; see Table 1):

HIP 78530 B. Was identified by Kouwenhoven et al. (2005) at a separation of $4.536 \pm 0.006^{\prime \prime}$ from the $\sim 2.5 M_{\odot}$ B9V star (Houk \& Smith-Moore 1988) HIP 78530 A. Lafrenière et al. (2011) confirmed the companion is co-moving with the primary star. The projected separation between the two objects now corresponds to $623 \pm 8$ au using the Gaia-DR2 distance (137.2 \pm 1.5 pc; Gaia Collaboration 2018) The Banyan $\Sigma$ tool (Gagné et al. 2018) and the DR2 astrometry confirms the star is a high-probability member $(99.9 \%)$ of the Upper-Scorpius association. Lafrenière et al. (2011) has provided a medium-resolution $\left(R_{\lambda} \sim 5300\right.$ to 6000) spectrum of the companion covering the $1.15-2.40 \mu \mathrm{m}$ wavelength range. Lachapelle et al. (2015) also presented a lower-resolution spectrum $\left(R_{\lambda} \sim 1350\right)$ but extending down to $1 \mu \mathrm{m}$. The spectra confirm the companion is a young M $7 \pm 0.5$ dwarf. Lachapelle et al. (2015) estimate a $T_{\text {eff }}$ of $2700 \pm 100 \mathrm{~K}$ and a luminosity of $\log \left(L / L_{\odot}\right)=-2.53 \pm 0.09$ relying on the HIPPARCOS distance available at that time. These latter authors find a mass of $23 \pm 2 M_{\text {Jup }}$ assuming an age of $10 \mathrm{Myr}$ for Upper-Scorpius. The primary star has no noticeable excess emission (Carpenter et al. 2009; Luhman \& Mamajek 2012; McDonald et al. 2017) and low extinction $\left(A_{v}=0.48\right.$ Neckel \& Klare 1980).

HIP $77900 B$. Was identified in UKIDSS and Pan-STARRS 1 images by Aller et al. (2013) from its red colors. It is located at a projected separation of 21.8" from the B6 star (Garrison 1967) HIP 77900, a high probability member of Upper-Sco (97.6\% membership probability according to Banyan $\Sigma$ ). Unlike for the case of HIP $78530 \mathrm{~B}$, the authors did not re-observe the target to check whether it shares the proper motion of the primary star. However, they obtained a low-resolution $\left(R_{\lambda} \sim 100\right)$ spectrum of the source covering the $0.8-2.5 \mu \mathrm{m}$ range whose features are indicative of a young M $9( \pm 0.5)$ object from Upper-Sco, and therefore argued that HIP $77900 \mathrm{~B}$ is bound to the star. We re-discuss the physical association of the two objects in Sect. 6.1.

USco 1610-1913B. Was identified by Kraus \& Hillenbrand (2008) and confirmed to be co-moving with the K7 star GSC 06209-00691 (Kraus \& Hillenbrand 2008, 2009; Kraus et al. 2014). The companion was last found at $5.837 \pm 0.006^{\prime \prime}$ from the star, now corresponding to a separation of $779 \pm 9 \mathrm{au}$ at the Gaia-DR2 distance of the primary. Aller et al. (2013) presented a $0.8-2.5 \mu \mathrm{m}$ low-resolution $\left(R_{\lambda} \sim 100\right)$ spectrum of the object confirming its youth and substellar nature. These latter authors estimated a M9 \pm 0.5 spectral type and found the companion to be four times overluminous with respect to HIP $77900 \mathrm{~B}$ which shared the same spectral properties at this spectral resolution. The spectral type was confirmed by Lachapelle et al. (2015) from a medium-resolution $\left(R_{\lambda} \sim 5300\right.$ to 6000$)$ NIR $(1.15-2.40 \mu \mathrm{m})$ spectrum of the companion. These latter author find $T_{\text {eff }}$ in the range $2300-2700 \mathrm{~K}$ for the object using DRIFT-PHOENIX and BT-SETTL atmospheric models with a large spread in $T_{\text {eff }}$ values depending on the wavelength interval considered for the fit. Their analysis also confirms the over-luminosity of the object for the inferred temperature. However, the star had no measured parallax at that time, and the average distance of Upper-Sco from de Zeeuw et al. (1999) was assumed. A second companion (hereafter USco 1610-1913 Ab) was discovered at a projected separation of $19.4 \pm 0.3$ au (Kraus et al. 2008) from USco 1610-1913 A. USco 1610-1913 Ab has a mass of $103 \pm 24 M_{\text {Jup }}$ using the contrast reported in Kraus et al. (2008), the new Gaia-DR2 distance of USco 1610-1913 A (assuming it is unbiased; see Sect. 6.1), and the Baraffe et al. (2015) tracks at an age of 5-11 Myr.

The six young and isolated free-floating objects (see Table 2) were selected from the sample of Lodieu et al. (2008) lateM/early-L Upper-Sco brown dwarfs. These latter authors also report low-resolution $\left(R_{\lambda} \sim 1700\right)$ spectra over $1.15-2.50 \mu \mathrm{m}$ of the sources. Low-resolution $\left(R_{\lambda} \sim 1350\right)$ multi-epoch redoptical $(0.57-0.88 \mu \mathrm{m})$ spectra of these objects have also been collected by Lodieu et al. (2011). The spectra of USco J16102239 and USco J1607-2211 exhibit a $\mathrm{H}_{\alpha}$ line. No emission line was detected at that time in the spectra of the remaining objects. Dawson et al. (2013) and Luhman \& Mamajek (2012) do not find noticeable infrared excess (up to $22 \mu \mathrm{m}$ ) for USco J1610-2239, USco J1607-2211 or USco J1607-2242. This is not the case for USco J1606-2335 and USco 1608-2315. The former is found to have Spitzer [4.5] and WISE W2 photometry indicative of a disk excess (Luhman \& Mamajek 2012), and the latter is found to have a W1-W2 color suggestive of an excess (Dawson et al. 2013) but that excess is not confirmed by Luhman \& Mamajek (2012) using similar data. Gaia-DR2 distances of $128.5_{-12.4}^{+15.4} \mathrm{pc}$ and $119.3_{-15.4}^{+20.8} \mathrm{pc}$ are available for USco J1610-2239 and USco J16072211. Using Banyan $\Sigma$ (Gagné et al. 2018), we find that the two objects have a 99.6 and $94.9 \%$ chance, respectively, of belonging to Upper-Scorpius based on kinematics. USco J1608-2315 also has a Gaia-DR2 parallax value ( $\pi=4.5073 \pm 1.1874$ mas). The object is found at a greater distance than the typical Upper-Sco members and therefore Banyan $\Sigma$ estimates a lower probability of membership to the association (59.7\%). The large error of DR2 for this object leads us to doubt this probability, noting that the target has spectral features clearly indicative of a membership to Upper-Sco (e.g., Lodieu et al. 2008; Bonnefoy et al. 2014). To conclude, adaptive-optics imaging and sparse aperture masking observations (Biller et al. 2011; Kraus \& Hillenbrand 2012) of the sources exclude companions with similar masses down to $\sim 10$ au. 
Table 2. Observing log.

\begin{tabular}{|c|c|c|c|c|c|c|c|c|}
\hline Target & $\begin{array}{l}\text { Date } \\
\text { (yyyy-mm-dd) }\end{array}$ & $\begin{array}{l}\text { UT Start-Time } \\
\text { (hh:mm) }\end{array}$ & $\begin{array}{l}\text { DIT } \\
(\mathrm{s})\end{array}$ & NDIT & NEXP & $\begin{array}{l}\langle\text { Seeing }\rangle \\
\left({ }^{\prime \prime}\right)\end{array}$ & Airmass & Notes \\
\hline USco 161031.9-16191305 B & 2014-04-02 & $08: 45$ & $190 / 190 / 200$ & $1 / 1 / 1$ & $8 / 8 / 8$ & 0.75 & 1.02 & \\
\hline \multirow[t]{2}{*}{ HIP $77900 \mathrm{~B}$} & 2014-04-12 & $05: 45$ & $190 / 190 / 197$ & $1 / 1 / 1$ & $14 / 14 / 14$ & 1.14 & 1.03 & \\
\hline & 2014-04-12 & $08: 38$ & 190/190/197 & $1 / 1 / 1$ & $14 / 14 / 14$ & 1.01 & 1.10 & \\
\hline \multirow[t]{2}{*}{ HIP $78530 \mathrm{~B}$} & 2014-06-09 & 04:08 & $190 / 190 / 197$ & $1 / 1 / 1$ & $14 / 14 / 14$ & 0.50 & 1.03 & \\
\hline & 2014-06-09 & $05: 14$ & 190/190/197 & $1 / 1 / 1$ & $14 / 14 / 14$ & 0.85 & 1.15 & \\
\hline \multirow[t]{2}{*}{ USco J160723.82-221102.0 } & 2014-04-22 & 09:01 & $190 / 190 / 197$ & $1 / 1 / 1$ & $8 / 8 / 8$ & 0.73 & 1.20 & \\
\hline & 2014-06-14 & $23: 50$ & $190 / 190 / 197$ & $1 / 1 / 1$ & $14 / 14 / 14$ & 0.52 & 1.33 & \\
\hline \multirow[t]{4}{*}{ USco J160606.29-233513.3 } & $2014-06-25$ & $04: 13$ & 190/190/197 & $1 / 1 / 1$ & $14 / 14 / 14$ & 0.86 & 1.14 & \\
\hline & 2014-06-30 & 03:09 & $190 / 190 / 197$ & $1 / 1 / 1$ & $14 / 14 / 14$ & 0.69 & 1.06 & \\
\hline & 2014-07-04 & 01:05 & 190/190/197 & $1 / 1 / 1$ & $16 / 16 / 16$ & 0.65 & 1.02 & \\
\hline & 2014-07-29 & $00: 44$ & $190 / 190 / 197$ & $1 / 1 / 1$ & $16 / 16 / 16$ & 0.67 & 1.03 & \\
\hline \multirow[t]{3}{*}{ USco J161047.13-223949.4 } & 2014-06-30 & $04: 21$ & $190 / 190 / 197$ & $1 / 1 / 1$ & $14 / 14 / 14$ & 0.88 & 1.20 & (1) \\
\hline & 2014-07-01 & $03: 26$ & $190 / 190 / 197$ & $1 / 1 / 1$ & $6 / 6 / 6$ & 1.03 & 1.06 & \\
\hline & 2014-07-01 & 04:04 & 190/190/197 & $1 / 1 / 1$ & $14 / 14 / 14$ & 1.14 & 1.16 & \\
\hline \multirow[t]{5}{*}{ USco J160737.99-224247.0 } & 2014-07-04 & 05:07 & $190 / 190 / 197$ & $1 / 1 / 1$ & $14 / 14 / 14$ & 1.38 & 1.51 & \\
\hline & 2014-07-29 & 02:07 & 190/190/197 & $1 / 1 / 1$ & $14 / 14 / 14$ & 0.61 & 1.16 & \\
\hline & 2014-07-29 & 03:08 & $190 / 190 / 197$ & $1 / 1 / 1$ & $14 / 14 / 14$ & 0.54 & 1.39 & \\
\hline & 2014-08-02 & $01: 49$ & $190 / 190 / 197$ & $1 / 1 / 1$ & $16 / 16 / 16$ & 1.33 & 1.16 & \\
\hline & 2014-08-02 & 03:09 & 190/190/197 & $1 / 1 / 1$ & $16 / 16 / 16$ & 1.12 & 1.51 & \\
\hline \multirow[t]{4}{*}{ USco J160818.43-223225.0 } & 2014-07-02 & $03: 16$ & $190 / 190 / 197$ & $1 / 1 / 1$ & $14 / 14 / 14$ & 1.11 & 1.07 & \\
\hline & 2014-07-02 & $04: 26$ & 190/190/197 & $1 / 1 / 1$ & $14 / 14 / 14$ & 0.80 & 1.25 & \\
\hline & 2014-07-03 & $03: 24$ & $190 / 190 / 197$ & $1 / 1 / 1$ & $14 / 14 / 14$ & 1.03 & 1.10 & \\
\hline & 2014-07-04 & $03: 49$ & $190 / 190 / 197$ & $1 / 1 / 1$ & $14 / 14 / 14$ & 1.14 & 1.16 & \\
\hline \multirow[t]{2}{*}{ USco J160828.47-231510.4 } & 2014-06-15 & 01:00 & $190 / 190 / 197$ & $1 / 1 / 1$ & $14 / 14 / 14$ & 0.95 & 1.11 & \\
\hline & 2014-06-20 & 05:06 & 190/190/197 & $1 / 1 / 1$ & $14 / 14 / 14$ & 1.20 & 1.23 & \\
\hline
\end{tabular}

Notes. The seeing is measured at $0.5 \mu \mathrm{m}$ and given for the visible arm. The DIT (Detector Integration Time) values refer to the individual exposure time per frame in the UVB, VIS, and NIR arms, respectively. NDIT are the number of individual frames per exposure, and $N_{\mathrm{EXP}}$ the number of exposures in the UVB, VIS, and NIR arms. ${ }^{(1)}$ no STD observed.

The optical and NIR spectra of the free-floating sources presented here have already been briefly introduced in Lodieu et al. (2018). These sources have increased spectral resolution or are extended at shorter wavelengths compared to previous spectroscopic data, and thus allow us to study the long-term variability of the $\mathrm{H}_{\alpha}$ line. We therefore re-analyze them in order to use them as empirical templates for the characterization of the three companions HIP 78530 B, HIP 77900 B, and USco 1610-1913 B.

\section{Observations and data reduction}

We used the X-shooter seeing-limited medium-resolution spectrograph mounted at UT2 Cassegrain focus (Vernet et al. 2011). The wide wavelength coverage of the instrument $(300-2480 \mathrm{~nm})$ is ideally suited for the characterization of accreting BDs with emission line series. We chose the $1.6^{\prime \prime}, 1.5^{\prime \prime}$, and $1.2^{\prime \prime}$-wide slits for the UVB, VIS, and NIR arms corresponding to resolving powers $R_{\lambda}=\lambda / \Delta \lambda=3300,5400$, and 4300, respectively. This setup was adopted for all our targets. The observing log is reported in Table 2. The slits were oriented perpendicular to the position angles of the companions in order to mitigate the flux contamination of the host stars. Each target was observed following an ABBA strategy to evaluate and remove the sky emission at the data processing step. Spectrophotometric standard stars were observed as part of the observatory calibration plan; they are not reported in the $\log$ as they were not used for our reduction or analysis.

We used the ESO reflex data-reduction environment (Freudling et al. 2013) to run the $\mathrm{X}$-shooter pipeline version 2.9.3 on the raw data (Modigliani et al. 2010). The pipeline produces two-dimensional, curvature-corrected, and flux-calibrated spectra for each target and epoch of observation (trace). The spectra were extracted from the traces using a custom IDL script. The flux in each wavelength channel at the position of the source was averaged within 720 mas aperture in the UVB and VIS arms, and a 1120 mas aperture in the NIR arm. The script computed the noise at the position of the source into each spectral channel following the procedure described in Delorme et al. (2017a). The residual nonlinear pixels in the spectra were removed using the kappa-sigma clipping method. The telluric corrections were evaluated and removed using the molecfit package (Smette et al. 2015; Kausch et al. 2015). The spectra at each epoch were corrected from the barycentric velocity and renormalized using the epochs when the sky transmission was photometric as an anchor point. Our flux calibration was checked by computing the 2MASS or MKO synthetic photometry from the spectra and comparing the values to published ones (Lodieu et al. 2008).

\section{Physical properties}

\subsection{Empirical analysis}

Lachapelle et al. (2015) derived the spectral types of USco 16101913 B and HIP 78530 B using NIR spectra of these companions. We noticed significant differences between them and the X-shooter spectrum of HIP 78530 B presented here (see Fig. 1). We noticed the same difference for the same target in comparing with the spectrum from Lafrenière et al. (2011). The X-shooter 


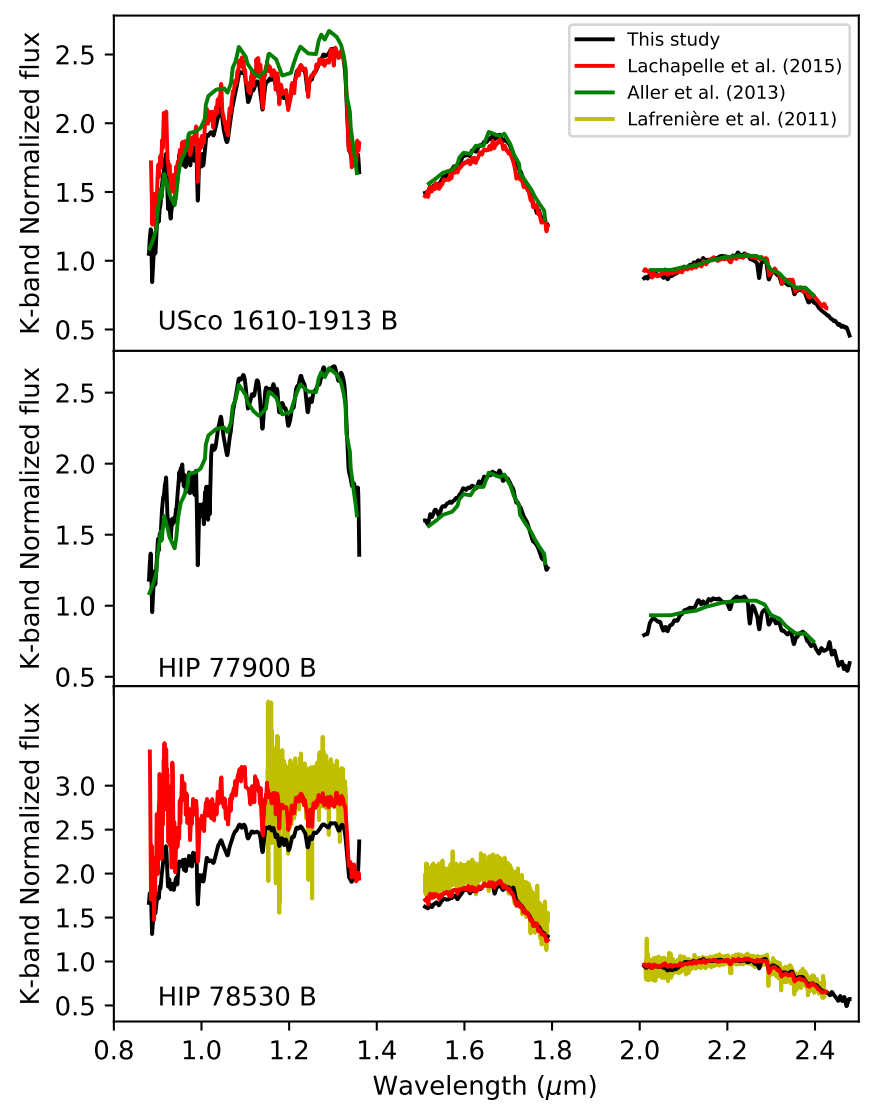

Fig. 1. Comparison between our spectra (black from X-shooter), those from Lachapelle et al. (2015) in red $(R=900)$ and Aller et al. (2013) in green (digitized), and those from Lafrenière et al. (2011) in yellow $(R=5300-6000)$.

spectrum of USco 1610-1913 B is consistent with the lowresolution spectrum of Aller et al. (2013). We therefore conclude that the differences in the $J$-band spectrum of HIP $78530 \mathrm{~B}$ may stem from flux losses affecting long-slit observations with AO-fed spectrographs.

\subsubsection{Line identification}

Figures 2 and 3 show the UVB+optical, $J-, H$-, and $K$-band segments of the X-shooter spectra of HIP 78530 B, HIP 77900 B, and USco 1610-1913 B together with two young, isolated brown dwarfs USco 1607-2211 (M 8.5) and TWA 26 (M 9). In the optical part, one can easily identify in Fig. 2 (left) the detection of the strong $\mathrm{H}_{\alpha}$ line $(0.6563 \mu \mathrm{m})$. Additional Balmer lines, $\mathrm{H}_{\gamma}(0.4340 \mu \mathrm{m})$ and $\mathrm{H}_{\beta}(0.4861 \mu \mathrm{m})$, are also detectable in the X-shooter spectra of USco 1610-1913 B and HIP 77900 B (also HIP $78530 \mathrm{~B}$ ) indicating the possible presence of accretion or chromospheric activity. The doublet of Ca II-H/K (0.3934, $0.3969 \mu \mathrm{m})$ is also detected. The detection of these emission lines is discussed in Sect. 5.

In $J$-band, we identified several strong absorption lines, like the neutral sodium ( $\mathrm{Na} \mathrm{I})$ doublet (1.138 and $1.141 \mu \mathrm{m})$, and the neutral potassium (K I) doublets $(1.168,1.177 \mu \mathrm{m}$ and 1.243, $1.254 \mu \mathrm{m})$. Neutral iron (Fe I) lines (1.189 and $1.197 \mu \mathrm{m})$ are also present as well as weaker Na I $(1.268 \mu \mathrm{m})$, magnesium (Mn I at $1.290 \mu \mathrm{m}$ ), and titanium ( $\mathrm{Ti}$ I at $1.283 \mu \mathrm{m}$ ) lines. We notice the typical triangular shape of the $H$-band which is produced by wide $\mathrm{H}_{2} \mathrm{O}$ absorption bands and testifies to the young ages of our objects. In $K$-band we found the calcium (Ca I) triplet (at
$1.98 \mu \mathrm{m})$ and a weak Na I doublet $(2.206,2.209 \mu \mathrm{m})$. We also detect CO bands $(2.295,2.322,2.352 \mu \mathrm{m})$.

\subsubsection{Spectral type and surface-gravity determination}

We re-investigated the optical classification of our targets using a standard $\chi^{2}$ comparison of our spectra to empirical templates from the Ultracool RIZzo Spectral Library ${ }^{1}$. The RIZzo library is made of spectra of 265 M2-L5 brown dwarfs from Cruz et al. (2003), Cruz et al. (2007), and Reid et al. (2008). We restrained the fit to the $0.75-0.86 \mu \mathrm{m}$ range. The results of our spectral fitting are shown in Fig. 4 and are reported in Table 3. The spectral type errors are due to the subgroup increment defined in the library. For USco 1610-1913 B, HIP 77900 B, and HIP 78530 B, we find spectral types of $\mathrm{M} 9 \pm 0.5, \mathrm{M} 9 \pm 0.5$, and $\mathrm{M} 8 \pm 0.5$, respectively, with best fits 2MASS J11582484+1354456 (M9) and 2MASS J07140394+3702459 (M 8) which are both freefloating objects (determined from BANYAN $\Sigma$ Tool). We also classified our targets in the NIR using absorption lines respecting the Allers \& Liu (2013) scheme. In NIR, we find later spectral types for USco 1610-1913 B and HIP 78530 B than the ones derived by Lachapelle et al. (2015), consistent with the redder slope of the X-shooter spectrum of HIP $78530 \mathrm{~B}$, and because of the revised extinction $A_{\mathrm{v}}$ values (see also Sect. 4.3) considered for the two systems. The optical spectral types derived for the free-floating BDs are in agreement with those of Lodieu et al. (2018) within error bars.

Appendix B shows the systematic differences between each method. The spectral type derived from the $\mathrm{H}_{2} \mathrm{O}$ index seems to be one to two subtypes over the one from the visual method. Allers \& Liu (2013) explain that the $\mathrm{H}_{2} \mathrm{O}$ index could be sensitive to gravity and so can be biased. We choose the spectral type from the visual comparison to avoid this bias.

In addition to the spectral-type determination, we also applied the surface-gravity classification formalized by Allers \& Liu (2013). The results are reported in Table 3 and show that HIP $77900 \mathrm{~B}$ and HIP $78530 \mathrm{~B}$ are identified as young, intermediate-surface-gravity BDs. USco 1610-1913 B is confirmed as a very-low-surface-gravity BD. The gravity class of HIP 78530 B is consistent with that derived by Lachapelle et al. (2015). Our results are consistent with those of Lodieu et al. (2018) for the classification of the young free-floating objects.

\subsubsection{Over-luminosity of USco 1610-1913 B}

Figure 5 shows the comparison between the visible and NIR calibrated flux of USco 1610-1913 B with the fluxes of young reference BDs of similar spectral types from our sample with Gaia parallaxes. We also include a comparison to the X-shooter spectrum of TWA 26 (Manara et al. 2013). All BDs have been scaled to the distance of USco 1610-1913A. All these objects reproduce the detailed absorptions and pseudo-continuum shape of USco 1610-1913 B spectrum provided that an extra scaling factor of two (TWA 26) and four (USco 1610-2239, HIP 77900 B) is considered. This over-luminosity of USco 1610-1913 B has already been noted by Aller et al. (2013) and Lachapelle et al. (2015). We confirm it over our extended wavelength range and resolution relying on an extended set of comparison objects from the association with now-published parallaxes. We discuss the possible origins of the over-luminosity in Sect. 6.

\footnotetext{
1 https://jgagneastro. com/the-ultracool-rizzospectral-library/
} 

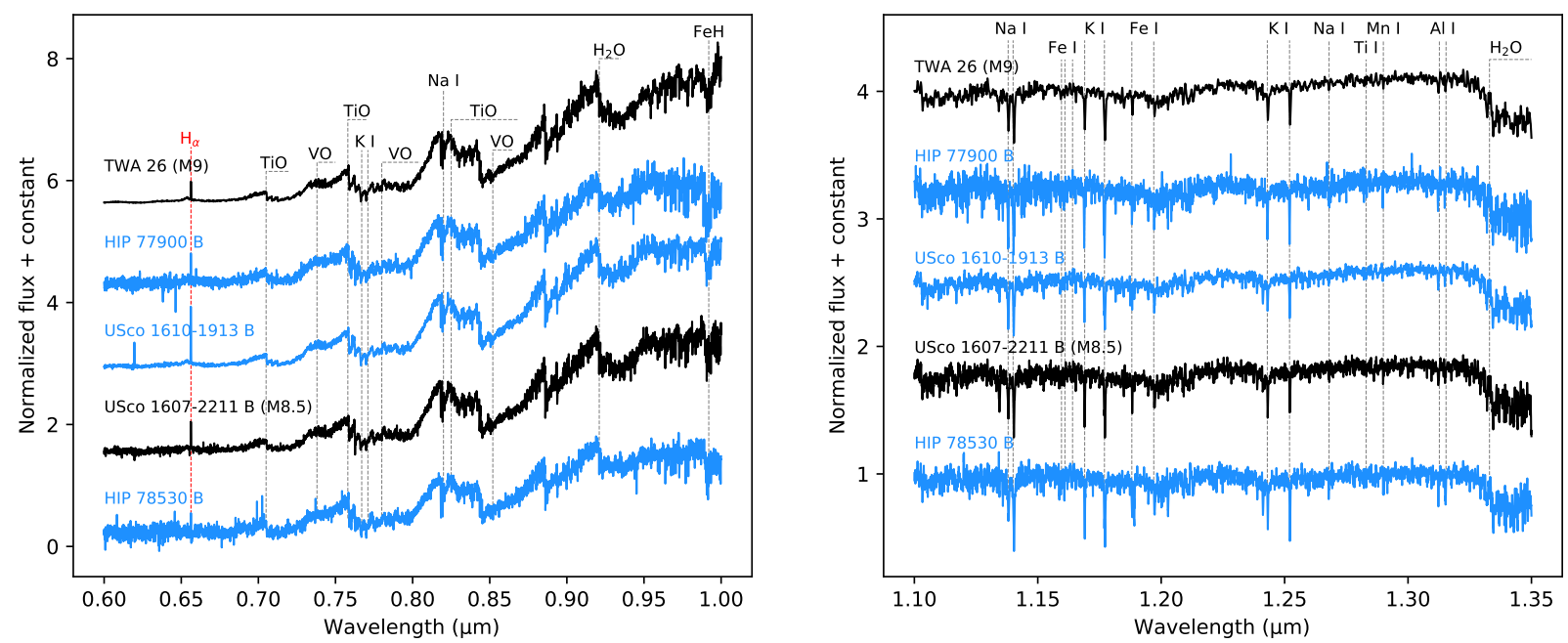

Fig. 2. Comparison of the optical spectra normalized at $0.82 \mu \mathrm{m}$ (left) and $J$-band normalized at $1.32 \mu \mathrm{m}$ (right) of USco 1610-1913 B, HIP 77900 B, and HIP 78530 B (blue) to the free-floating object USco 1607-2211 from our original sample of spectra of Upper-Scorpius BDs and to the TW Hydrae association member TWA 26 (Manara et al. 2013) (black). All objects have a $\mathrm{H}_{\alpha}$ emission line (656.3 nm; red label) in addition to molecular and atomic absorption lines typical of late-M dwarfs.
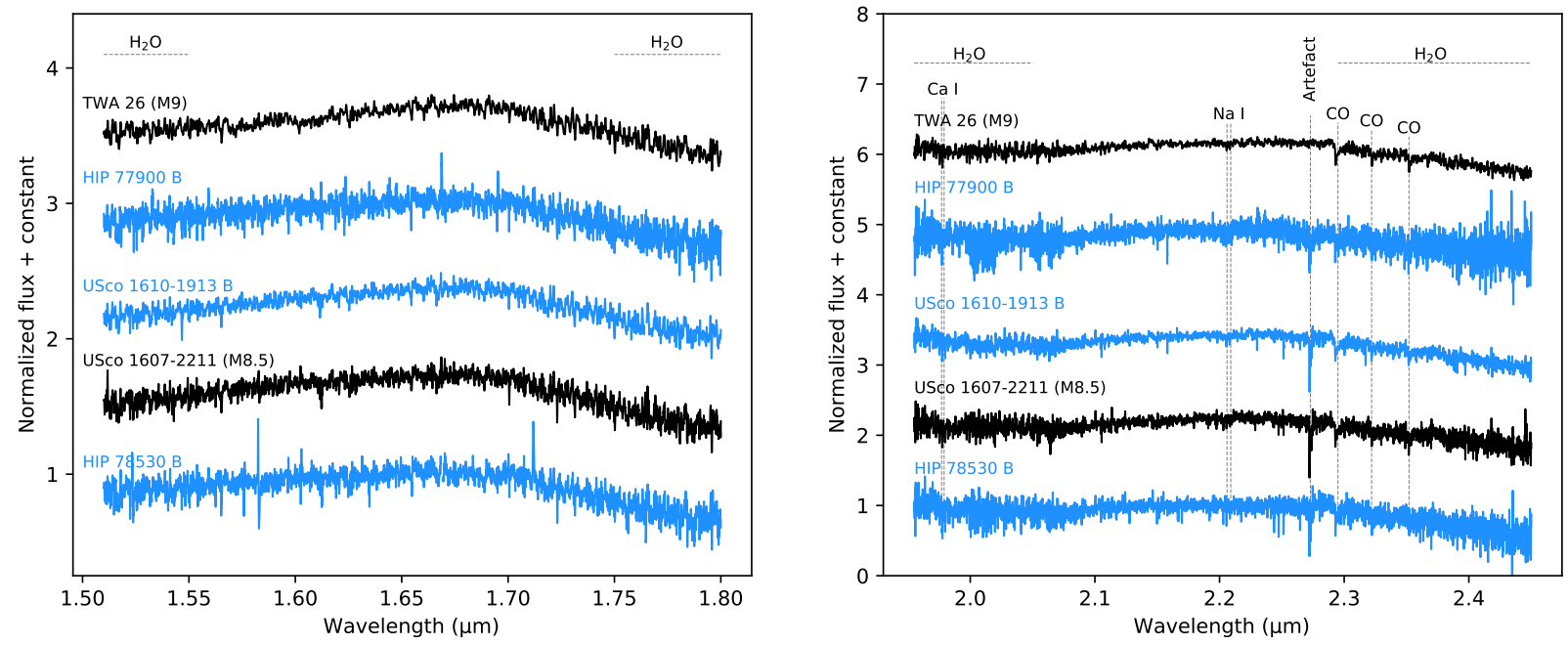

Fig. 3. As in Fig. 2 but for $H$-band normalized at $1.65 \mu \mathrm{m}$ (left) and $K$-band normalized at $2.15 \mu \mathrm{m}$ (right).

Table 3. Results of the empirical analysis.

\begin{tabular}{|c|c|c|c|c|c|c|c|c|c|}
\hline \multirow{3}{*}{ Source } & \multicolumn{4}{|c|}{ Spectral types } & \multicolumn{5}{|c|}{ Gravity } \\
\hline & \multirow[t]{2}{*}{ Visual } & \multicolumn{3}{|c|}{ NIR } & \multicolumn{4}{|c|}{ Score ${ }^{(a)}$} & \multirow[t]{2}{*}{ Type } \\
\hline & & $\mathrm{H}_{2} \mathrm{O}(\mathrm{H})^{(b)}$ & $\mathrm{H}_{2} \mathrm{O}-1(\mathrm{~J})^{(c)}$ & $\mathrm{H}_{2} \mathrm{O}-2(\mathrm{~K})^{(c)}$ & $\mathrm{FeH}_{z}$ & $\mathrm{FeH}_{j}$ & $\mathrm{~K} \mathrm{I}_{j}$ & H-cont & \\
\hline USco 1610-1913 B & $\mathrm{M} 9 \pm 0.5$ & M9.1 \pm 0.2 & $\mathrm{~L} 0.1 \pm 0.2$ & $\mathrm{M} 8.9 \pm 0.2$ & 2 & 2 & 2 & 2 & v-low \\
\hline HIP $77900 \mathrm{~B}$ & $\mathrm{M} 9 \pm 0.5$ & $\mathrm{M} 8.4 \pm 0.5$ & M9.9 \pm 0.4 & M9.5 \pm 0.5 & 1 & 2 & 1 & 2 & int \\
\hline HIP 78530 B & $\mathrm{M} 8 \pm 0.5$ & M8.4 \pm 0.4 & M8.4 \pm 0.4 & $\mathrm{M} 8.2 \pm 0.4$ & 1 & 2 & 1 & 2 & int \\
\hline USco 1607-2242 & $\mathrm{M} 9 \pm 0.5$ & $\mathrm{~L} 1.1 \pm 1.9$ & $\mathrm{~L} 1.7 \pm 1.5$ & $\mathrm{~L} 0.7 \pm 1.7$ & $\mathrm{n}$ & 1 & 2 & 2 & v-low \\
\hline USco 1608-2232 & $\mathrm{M} 9 \pm 0.5$ & $\mathrm{~L} 0.9 \pm 0.8$ & $\mathrm{~L} 1.5 \pm 0.6$ & $\mathrm{~L} 0.1 \pm 0.7$ & 2 & 2 & 1 & 2 & v-low \\
\hline USco 1606-2335 & M9 \pm 0.5 & M9.9 \pm 0.9 & $\mathrm{~L} 0.5 \pm 0.8$ & M9.3 \pm 1.0 & 2 & 1 & 1 & 2 & int \\
\hline USco 1610-2239 & $\mathrm{M} 9 \pm 0.5$ & M9.9 \pm 0.5 & $\mathrm{~L} 0.3 \pm 0.4$ & M9.3 \pm 0.5 & 2 & 2 & 2 & 2 & v-low \\
\hline USco 1608-2315 & M8.5 \pm 1.0 & $\mathrm{~L} 0.0 \pm 0.4$ & $\mathrm{~L} 0.6 \pm 0.4$ & M9.1 \pm 0.4 & 2 & 2 & 1 & 2 & v-low \\
\hline USco 1607-2211 & M8.5 \pm 1.0 & M9.4 \pm 0.5 & $\mathrm{~L} 0.4 \pm 0.4$ & $\mathrm{M} 8.3 \pm 0.5$ & 2 & 2 & 1 & 2 & v-low \\
\hline
\end{tabular}

Notes. The systematic differences about the spectral-type determination are discussed in Appendix B.

References. Relations and coefficients: ${ }^{(a)}$ Allers \& Liu (2013), ${ }^{(b)}$ Allers et al. (2007), ${ }^{(c)}$ Slesnick et al. (2004). 


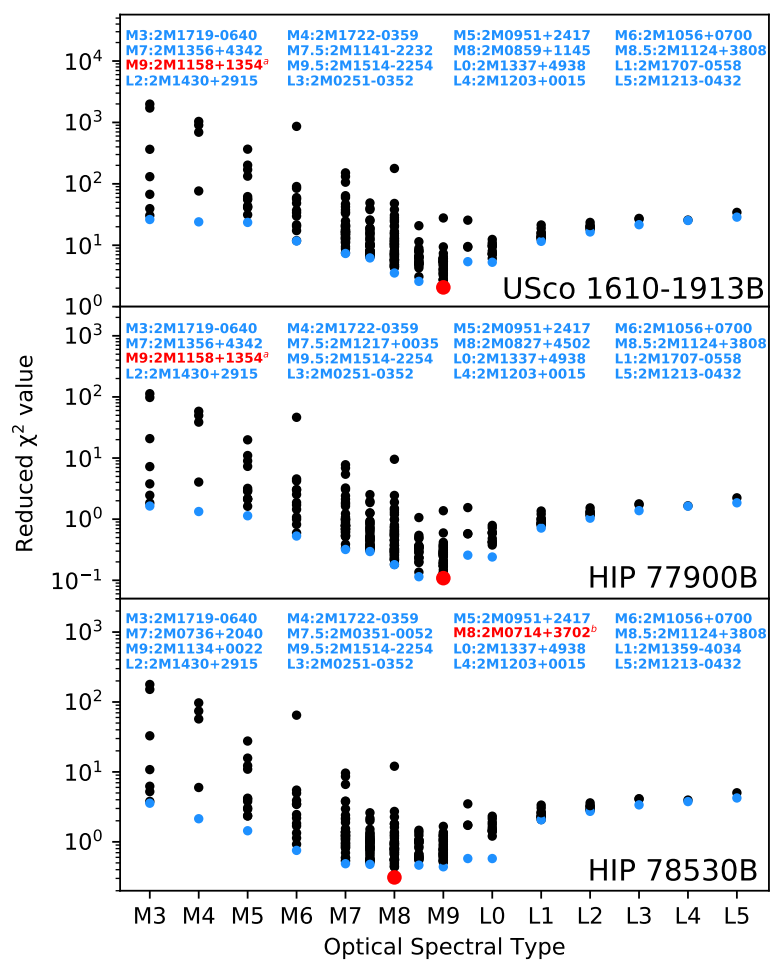

Fig. 4. Chi-squared from the comparison between the X-shooter spectra of USco 1610-1913 B, HIP 77900 B, and HIP 78530 B to the optical spectra of the Ultracool RIZzo spectral library. We indicate the bestfitting object for each group in blue. The $\chi^{2}$ minimum for all objects is represented in red. (a) : Cruz et al. (2003). (b) : Reid et al. (2008).

\subsection{Forward modeling analysis}

\subsubsection{Description of the atmospheric models}

We used a forward-modeling approach to determine the atmospheric parameters of the young BDs observed in this study. Forward modeling codes enable comparison of the object spectrum to pre-computed grids of models which include our best knowledge of atmospheric physics. We used grids of synthetic spectra produced by the BT-SETTL15 model (Allard et al. 2012). This model handles the radiative transfer using the PHOENIX code (Hauschildt et al. 1997; Allard et al. 2001). It accounts for convection using the mixing-length theory, and works at hydrostatic and chemical equilibrium. The opacities are treated line by line (details on each element are given in Rajpurohit et al. 2018). The code models the condensation, coalescence, and mixing of 55 types of grains. The abundances of solids are determined comparing the timescales of these different processes at each layer. In this study, we considered the predictions of the BT-SETTL15 model with $T_{\text {eff }}$ ranging from 2100 to $3000 \mathrm{~K}$ (in steps of $100 \mathrm{~K}$ ), and a range of $\log (\mathrm{g})$ from 3.5 to $5.5 \mathrm{dex}$ (in steps of $0.5 \mathrm{dex}$ ). We assumed a solar metallicity $M / H=0.0$, in broad agreement with the values reported in Sco-Cen (Bubar et al. 2011). These grids have been extensively used in previous studies of young BDs (Manjavacas et al. 2014; Bonnefoy et al. 2014; Delorme et al. 2017b; Bayo et al. 2017), but never as part of a Bayesian methodology, as developed here with the ForMoSA code.

\subsubsection{The ForMoSA code}

For this work, we chose to develop our own forward modeling code relying on the Nested Sampling procedure (Skilling 2006).

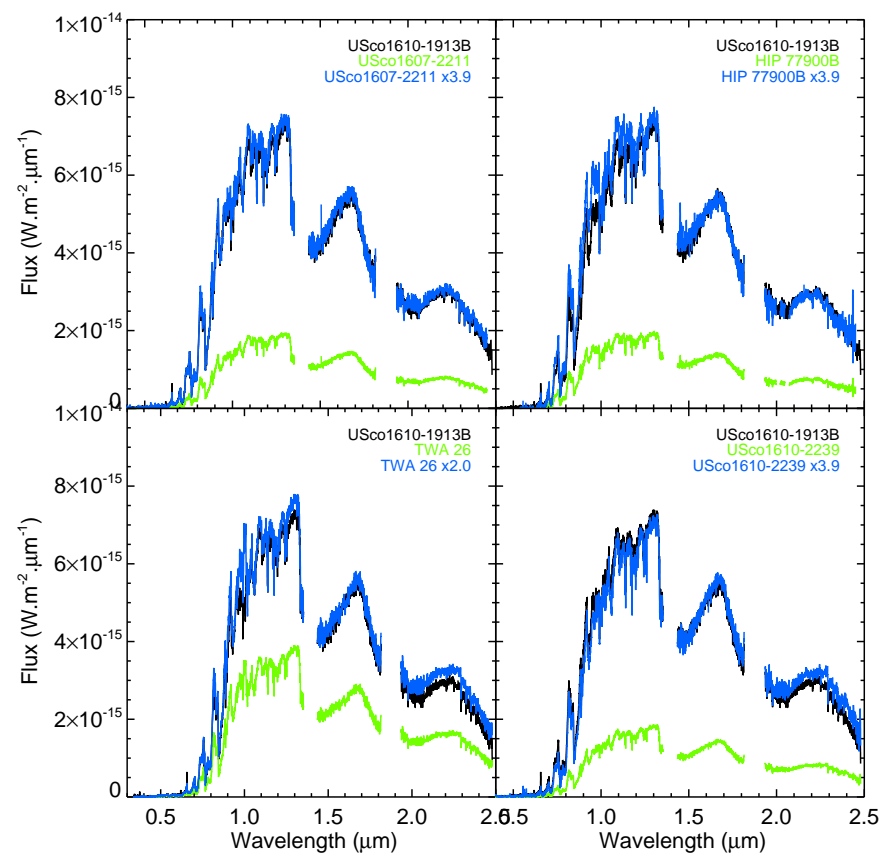

Fig. 5. Comparison of the flux-calibrated $0.4-2.5 \mu \mathrm{m}$ spectra of USco 1610-1913 B (black) to those of reference objects scaled to the distance of the companion (green). The companion is 2.0-3.9 times more luminous than objects with the same spectral type.

The method explores and recursively isolates different patches of likelihood levels in the parameter space. It offers several advantages over classical Markov-chain-Monte-Carlo algorithms also used for the forward modeling (e.g., Bonnefoy et al. 2018; Samland et al. 2017). The approach avoids missing local minimum within vast and degenerate parameter spaces while ensuring the convergence of the exploration.

The code ForMoSA (for FORward MOdeling for Spectral Analysis) takes as input an observed spectrum with associated error bars and any grid of synthetic spectra (here the BT-SETTL15 grid). The Nested Sampling is handled by the nestle Python module ${ }^{2}$. ForMoSA re-samples the data and the models in order to make them comparable. The grid of model spectra are first interpolated onto the wavelength grid of the observation and degraded to the spectral resolution of the instrument that acquired the data. The optical spectra of our targets and the models were degraded to $R_{\lambda}=3300,5400$ and 4300, similar to the UVB, VIS, and NIR parts of the spectrum, respectively.

In our case, we hypothesize that data points are independent in the observed spectra, and therefore the likelihood is derived from the $\chi^{2}$ value. To compute $\chi^{2}$ at each step, we need to generate a model spectrum for a set of free-parameters that does not necessarily exist in the original grid of spectra. Therefore, we generated a model spectrum on demand following a two-step process:

A preliminary phase consists in reducing the grid meshes. To do so, ForMoSA interpolates and reduces each $T_{\text {eff }}$ increment to $10 \mathrm{~K}$ and $\log (g)$ increments to $0.01 \mathrm{dex}$. We considered the linear and bicubic spline interpolation approaches, and finally selected the bicubic spline interpolation which better accounts for the flux variation through the grid. This step needs to be taken only once and ensures a regular grid. In doing this, we increase the accuracy of the second interpolation phase.

2 http://kylebarbary.com/nestle/ 


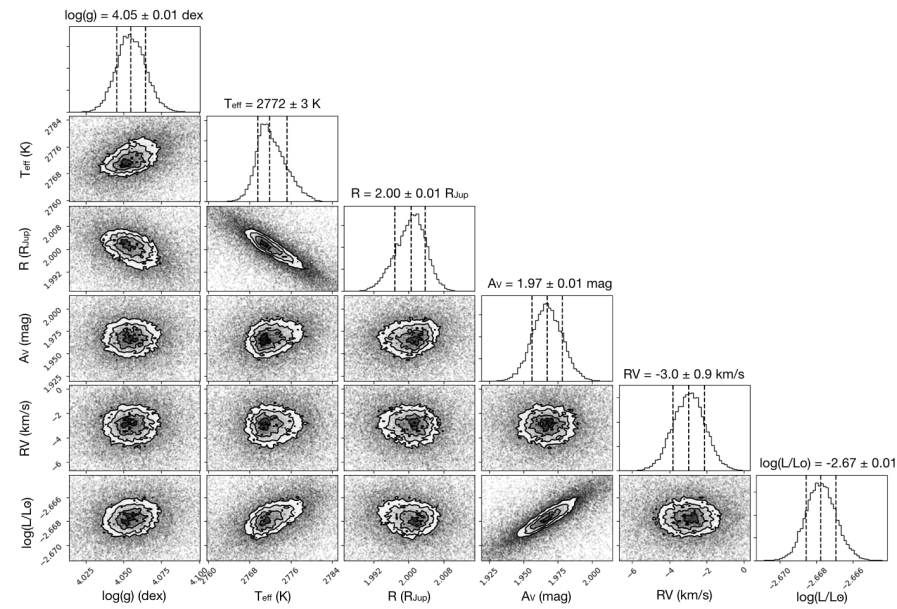

Fig. 6. Posteriors of HIP 78530 B in using ForMoSA on the $J+H+K$ and with the extinction parameter free.

The second phase arises in the course of the nested sampling process when a new point in the parameter space is defined. The closest neighbors approach was found to provide the best tradeoff between the reliability of the interpolation process and the computation time needed to run the interpolation.

Each synthetic spectrum gives the flux at the top of the atmosphere. The comparison with the observed spectrum also requires multiplication of the model by a dilution factor $C_{\mathrm{k}}=$ $\left(\frac{R}{d}\right)^{2}$, with $R$ the object radius and $d$ the distance. We adopt distances for the host stars from the Gaia DR2. We considered flat priors on $T_{\text {eff }}(2100-2900 \mathrm{~K})$ and $\log (g)(3.5-5.5 \mathrm{dex})$. Here, $T_{\text {eff }}$ and radius are linked together by the luminosity (Boltzmann law) so we have chosen a flat prior on $R\left(0.5-30.0 R_{\mathrm{Jup}}\right)$ to be conservative and ensure we do not limit the exploration of $T_{\text {eff }}$. We also provide the luminosity from the posterior distributions of $R$ and $T_{\text {eff }}$ ForMoSA can also consider interstellar extinction $\left(A_{\mathrm{v}}\right)$ as a free parameter using the extinction law from Fitzpatrick \& Massa (2007), the radial velocity (RV) by a Doppler shifting $\mathrm{law}^{3}$, and the projected rotational velocity $v \sin (i)$ according to the rotational broadening law from "The Observation and Analysis of Stellar Photospheres" by Gray ${ }^{4}$. Figure 6 illustrates a typical output of ForMoSA on HIP 78530 B. The method allows us to identify correlations between parameters such as $T_{\text {eff }}$ and $R$.

\subsection{Results}

As a safety check, the ForMoSA code was applied to our three companions HIP 78530 B, HIP 77900 B, and USco 16101913 B and two well-known young BDs, TWA 26 and TWA 29, with similar spectral types and ages ( $8 \mathrm{Myr})$ and observed by Manara et al. (2013). We considered wavelengths from 1.0 to $2.5 \mu \mathrm{m}$ in all our fits to avoid biases related to the residual contamination of HIP $78530 \mathrm{~B}$ and ran a homogeneous analysis on all objects. The results are shown in Figs. 7-11. The numerical values are given in Tables C.1-C.2.

Panels a-d of Figs. 7-11 show the X-shooter spectra (black) with the resulting best fit (blue) considering different spectral windows $(J+H+K, J, H$ and $K$, respectively), and a fixedextinction assumption from the Gaia DR2 extinction maps and values at the location of our targets (Lallement et al. 2019).

\footnotetext{
3 Function PyAstronomy.pyasl.dopplerShift

4 Function PyAstronomy.pyasl.rotBroad
}
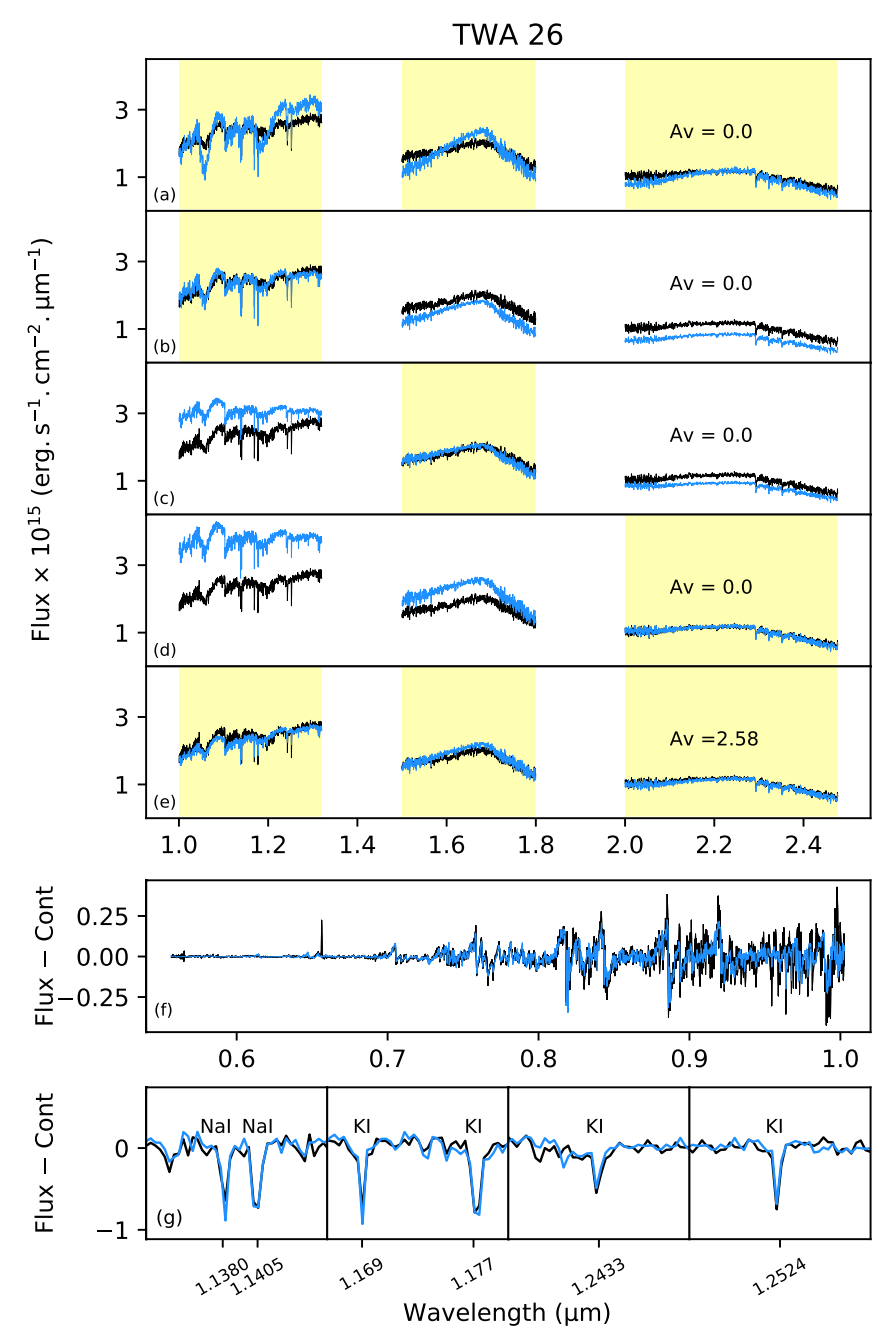

Fig. 7. Best fits of TWA 26 (in black) by BT-SETTL15 models (in blue) using different portions of the spectrum for the fit (shaded yellow rectangles). We define the fitting zones as follows: $J=1.0-1.32 \mu \mathrm{m}$; $H=1.5-1.8 \mu \mathrm{m} ; K=2.0-2.48 \mu \mathrm{m}$. We perform the following fits: (a) $J+H+K$; (b) $J$; (c) $H$; (d) $K$; (e) $J+H+K$ with Av as a free parameter; $(f)$ the optical part $(0.56-1.00 \mu \mathrm{m})$ of the flux-continuum and $v \sin (i)$ as a free parameter; and $(g)$ examples of lines fitted on the flux-continuum.

From these maps, we calculate the $A_{0}$ extinction at $550.0 \mathrm{~nm}$ and assume that the difference with the $A_{\mathrm{v}}$ extinction is negligible $\left(A_{0} \simeq A_{\mathrm{v}}\right)$. The fitting spectral range is indicated by the yellow background.

Panel e shows the best fit when using the complete $J+H+$ $K$-band spectral range, and adding the extinction value $A_{\mathrm{v}}$ this time as a free parameter. We reddened the models with an extinction function in the code (Fitzpatrick \& Massa 2007).

Panel $\mathrm{f}$ shows the best fit focused on the optical part (0.56-1.00 $\mu \mathrm{m}$ for all targets but $0.80-1.00 \mu \mathrm{m}$ for HIP $78530 \mathrm{~B})$. We estimate the pseudo-continuum from the original X-shooter spectrum by degrading it to a very low spectral resolution $\left(R_{\lambda}=100\right)$. We then subtract this pseudo-continuum from the original section to highlight line features. We add the $v \sin (i)$ as a free parameter with this fit. We determine the radius analytically with the relation from Cushing et al. (2008).

Panel g shows of zoomed view on a the gravity- and metallicity-sensitive lines of K I and $\mathrm{NaI}$ with the best-fitting solution. We subtracted the pseudo-continuum using the same 
TWA 29
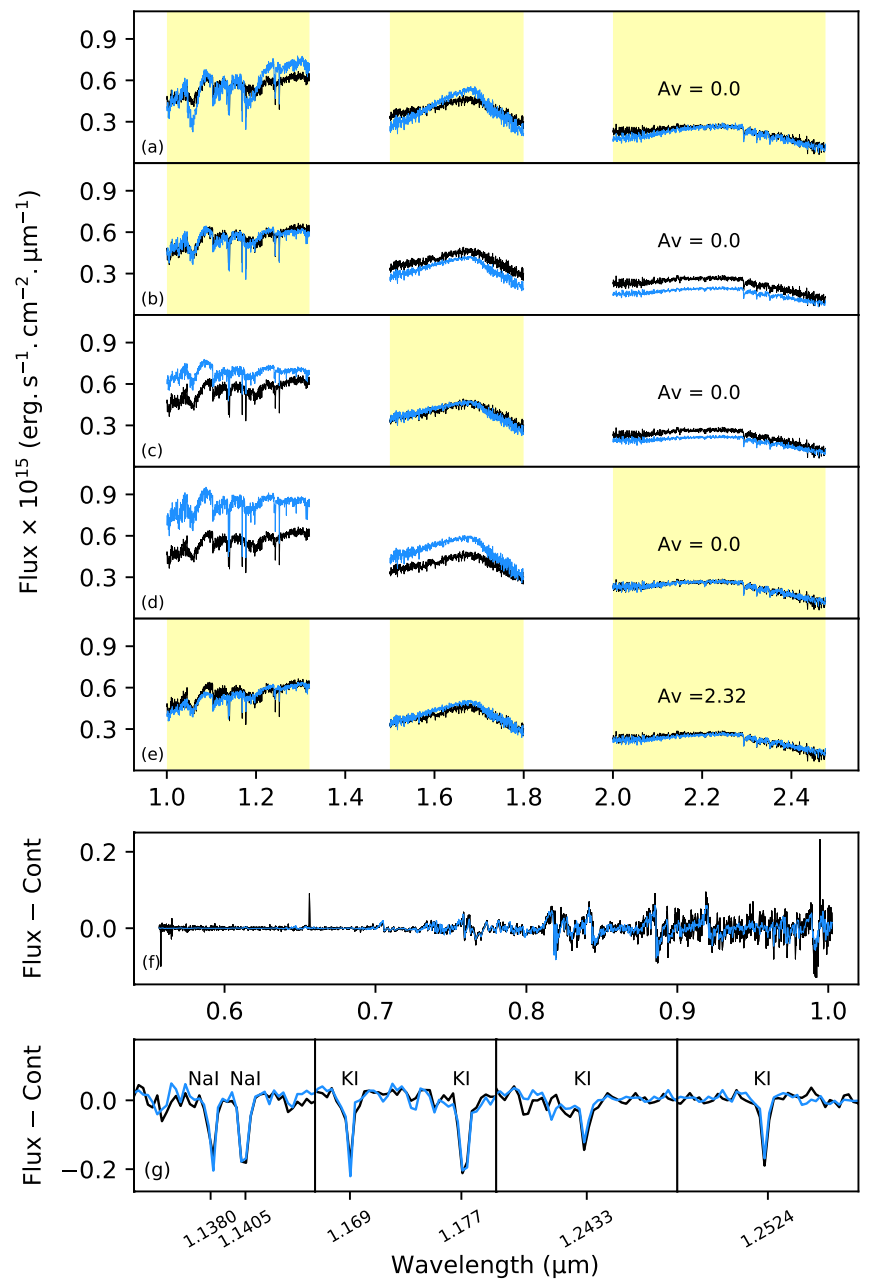

Fig. 8. Same as Fig. 7 but for TWA 29.

method as in panel $\mathrm{f}$. The determination of the radius is also analytic.

Generally, the results show that the BT-SETTL15 models fail to consistently reproduce the pseudo-continuum of the X-shooter spectra of all objects at all wavelengths (visible and NIR) with the same range of physical parameters. The same problem arises when considering a $\chi^{2}$ test on the original grid of BT-SETTL15 spectra. This is highlighted in panels a-d of all figures, where the best-fit solution varies considerably depending on which spectral range is fit. Similar discrepancies were evidenced by Manjavacas et al. (2014) using older releases of the models (Allard et al. 2011, 2012, 2013; Lachapelle et al. 2015; Bayo et al. 2017). Therefore, the problem remains in the 2015 release of the models.

The posteriors of $T_{\text {eff }}$ are strongly tied to the pseudocontinuum shape. Consequently, they are affected by the choice of the spectral range used to estimate the best fit. For instance, the $T_{\text {eff }}$ is generally higher from a fit using the $J$-band compared to a fit using $H$ - or $K$-band. The surface-gravity is also affected as it remains sensitive to the shape of the $\mathrm{VO}$ and $\mathrm{H}_{2} \mathrm{O}$ absorption bands. Notably, the radius, which is linked to the flux dilution factor, seems to be mostly consistent when using three different fitting bands. In addition to the best-fitting solution, the ForMoSA code provides the errors on the posterior solutions. The errors on the posterior solutions are tightly connected to the errors bars on the spectra themselves by the likelihood function. Given the high signal-to-noise ratio $(\mathrm{S} / \mathrm{N})$ of the $\mathrm{X}$-shooter spectra
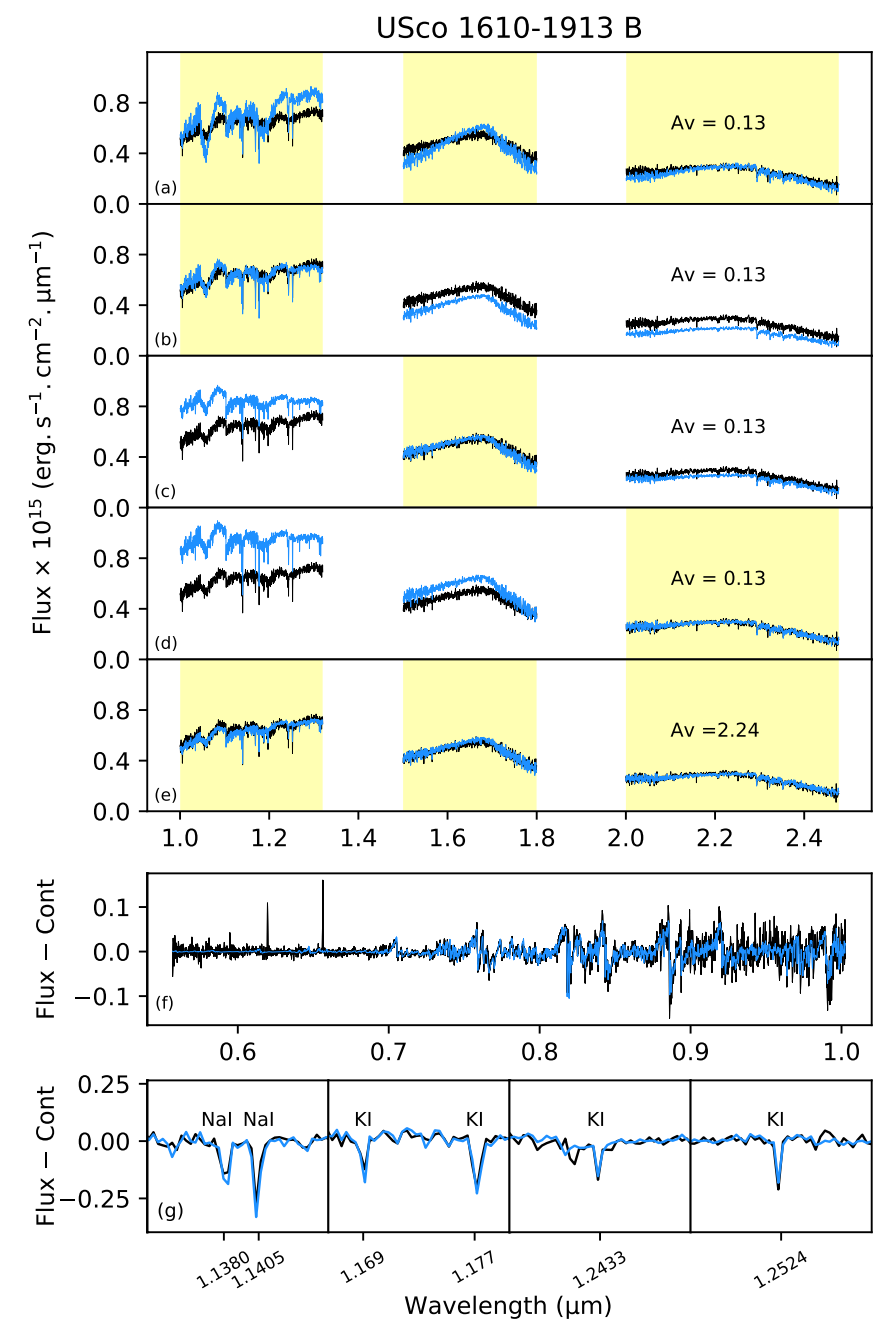

Fig. 9. Same as Fig. 7 but for USco 1610-1913 B.

for HIP 78530 B, HIP 77900 B, USco 1610-1913 B, TWA 26, and TWA 29, the resulting fitting error bars are about one to two orders of magnitude smaller than the systematic errors associated with the choice of the spectral fitting window and the non-perfect match of the BT-SETTL15 models with our spectra at all observed wavelengths (see Tables C.1-C.2). Our adopted solutions in $T_{\text {eff }}, \log (g)$, and $R$ are therefore derived from the average and dispersion of the solutions from the different spectral fitting windows with the extinction value given by Lallement et al. (2019). The bolometric luminosity is calculated with the Stefan-Boltzmann law.

In addition to the different spectral fitting window, the ForMoSA code was also applied with the extinction $A_{\mathrm{v}}$ as a free parameter. The resulting posteriors can be directly compared with the ones obtained with the Gaia DR2 values at the location of our targets (Lallement et al. 2019). Surprisingly, with the simple use of an interstellar extinction law correction, we considerably improve the goodness of the fits of all targets (see Figs. 7-11 panel e). Considering that our targets have no extinction (both sources are located at less than 80 pc; TWA 26 has a transition disk around it but the flux excess appears at $\lambda>20 \mu \mathrm{m}$, Riaz \& Gizis 2008; TWA 29 has no known disk, Rodriguez et al. 2015) or precisely known and low interstellar extinction values ( $A_{\mathrm{v}} \leq 0.13$ for our three Upper-Sco companions), the excellent good fit of all objects with artificial $A_{\mathrm{v}}$ values of $1.76-2.58 \mathrm{mag}$ indicates a missing physical component in the models. As all 

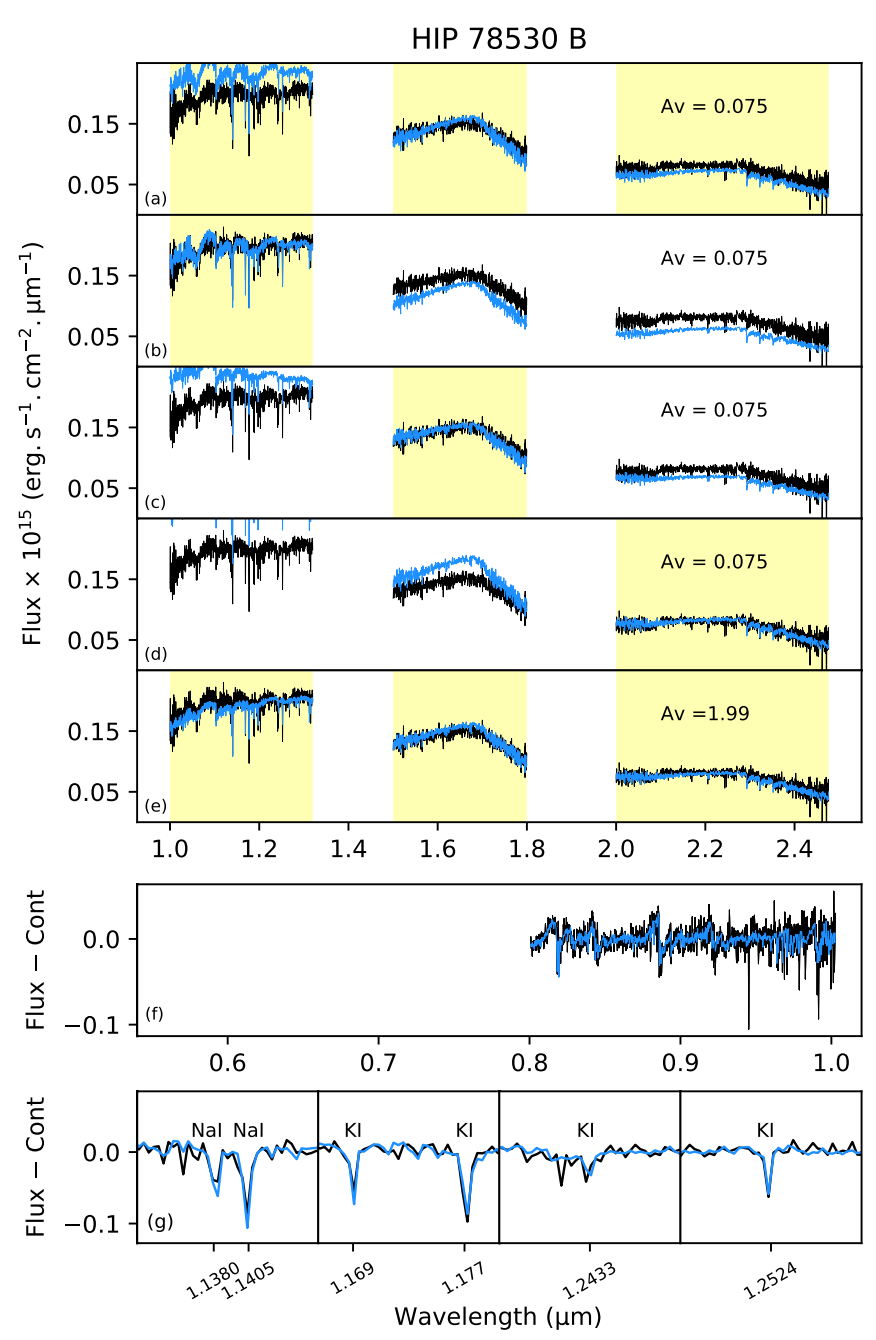

Fig. 10. Same as Fig. 7 but for HIP 78530 B. We avoid the flux inconsistency presented in Sect. 4.1 by using the wavelength range $0.8-1.0 \mu \mathrm{m}$ in panel $f$.

objects are affected, it is very likely that the origin is not circumstellar, but linked to the current state-of-the-art of BT-SETTL15 models, as is for example the complexity of the dust formation process or the dust opacity (Pavlenko et al. 2007). Systematically testing how this deficiency evolves with lower effective temperature, age (surface-gravity), and gravity would be very interesting.

As an additional step to exploit the medium resolution of our $\mathrm{X}$-shooter spectra, the radial and rotational velocity information (line profile and offset) has been incorporated into ForMoSA as additional fitting parameters. The RV can be adjusted on the full spectral window. For the adjustment of $v \sin (i)$, we restricted the fitting window to the optical part $(0.56-1.00 \mu \mathrm{m})$ with the continuum subtracted to minimize the computation time. This choice is driven by the higher spectral resolution in optical $(R=5400)$ and the presence of various absorption lines (NaI, FeH or VO). In Tables C.1 and C.2, one can see that the RV results strongly depend on the choice of the fitting spectral window. We adopted the RV value from the optical fit for the same reasons as for the $v \sin (i)$. The two identified causes of this discrepancy are the slope issues in models and a systematic error in the wavelength calibration of the spectra. We therefore decided to independently compute the RVs using a cross-correlation approach between our observed spectra and a spectral template generated by the
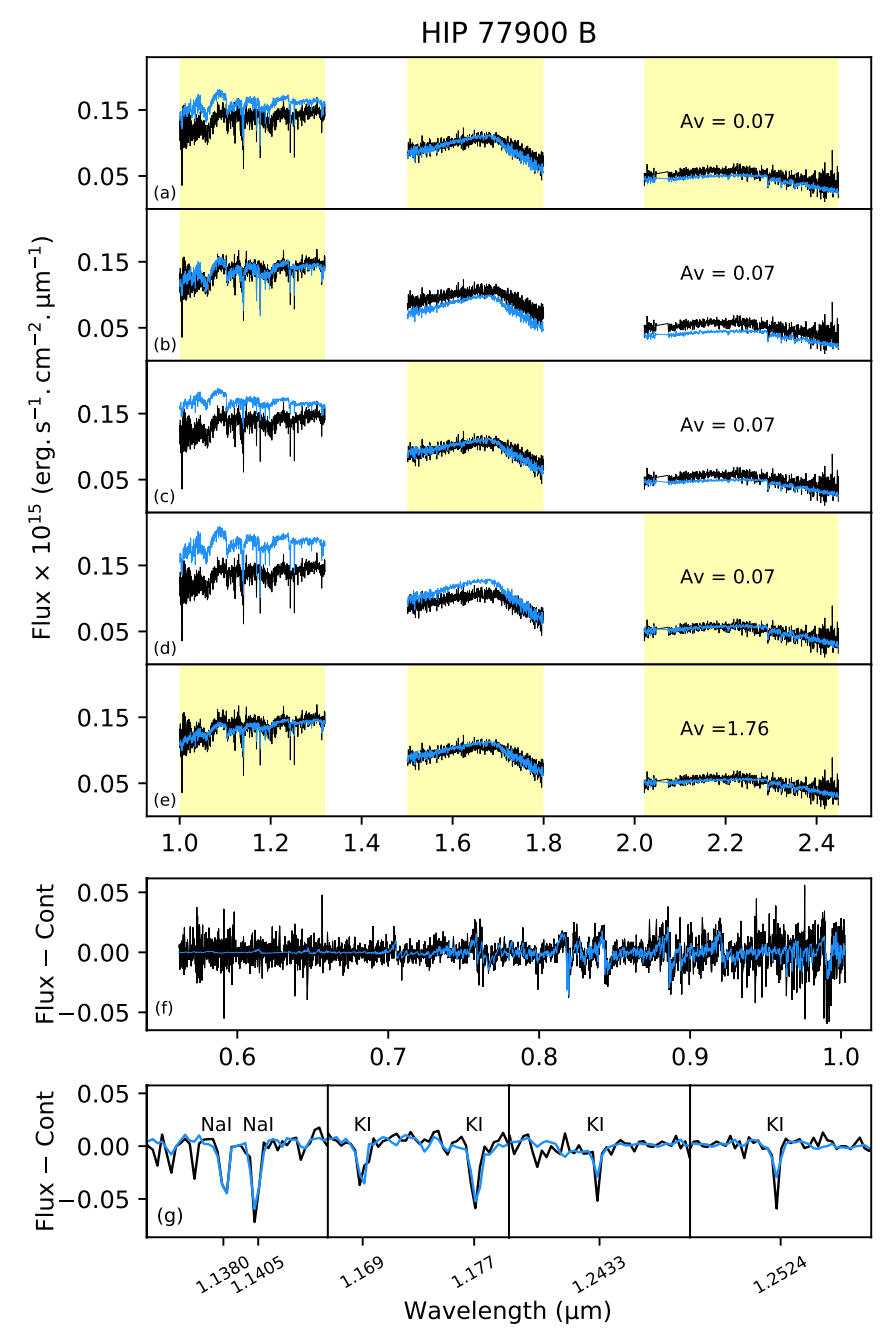

Fig. 11. Same as Fig. 7 but for HIP 77900 B.

BT-SETTL 15 code at $T_{\text {eff }}=2400 \mathrm{~K}$ and a $\log (g)=4.0$ dex (see Fig. 12). For most cases, there is a good agreement between the cross-correlation results and the ForMoSA ones in the optical that we therefore adopt as final values. For the rotational velocity, the limited $\mathrm{X}$-shooter spectral resolution does not allow the measurement of the low $v \sin (i)$ of our targets, but rather places an upper limit of roughly $\leq 50 \mathrm{~km} \mathrm{~s}^{-1}$. This limit is consistent with the $55 \mathrm{~km} \mathrm{~s}^{-1}$ limit expected for a resolution of about $R=5400$ in the visible arm. Considering the adopted values of $T_{\text {eff }}, \log (g), R, R V, v \sin (i)$ and $L$ using the fixed extinction values of Lallement et al. (2019), we now detail the results of ForMoSA target by target.

TWA26 and TWA29. Both targets are well known and their X-shooter spectra have been analyzed as a sanity check of the ForMoSA code. They have been classified by Manara et al. (2015) as late-M dwarfs with spectral types M9 $\gamma$ and M9.5, respectively. Both are members of the TW Hydrae association, with an age estimate of $8 \mathrm{Myr}$, and are located at a distance of $49 \mathrm{pc}$ and $83 \mathrm{pc}$, respectively. They have no extinction observed in their line of sight. Both were characterized by Filippazzo et al. (2015) using a complimentary approach to our work. Exploiting the spectral energy distribution from the visible to the NIR and the mid-infrared (MIR), Filippazzo et al. (2015) derived the bolometric luminosities of these two young brown dwarfs with known distances. Using evolutionary model 


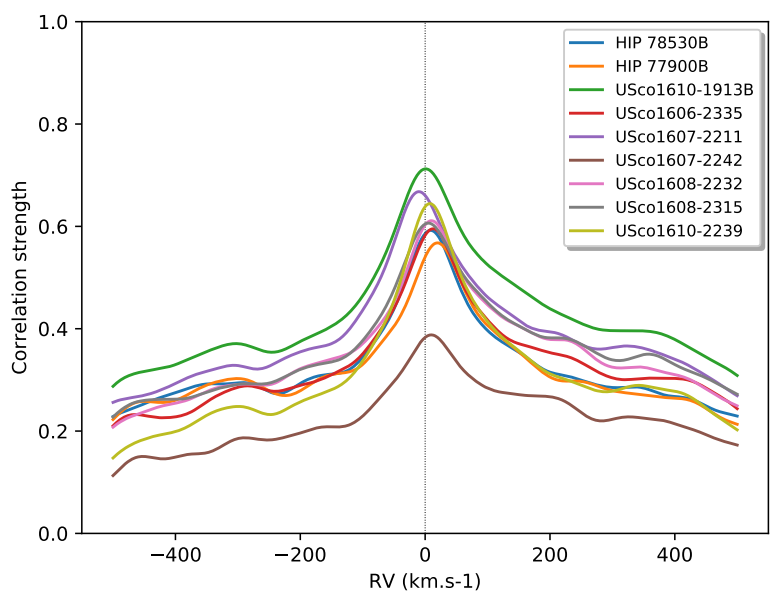

Fig. 12. Correlation strength as a function of RV for each of our objects. We use the synthetic spectra at $T_{\text {eff }}=2400 \mathrm{~K}$ and $\log (g)=4.0$ dex from BT-SETTL15.

Table 4. Physical properties of TWA 26 and TWA 29.

\begin{tabular}{cccccc}
\hline \hline & $\log \left(L / L_{\odot}\right)$ & $\begin{array}{c}R \\
\left(R_{\mathrm{Jup}}\right)\end{array}$ & $\begin{array}{c}\log (g) \\
(\mathrm{dex})\end{array}$ & $\begin{array}{c}T_{\text {eff }} \\
(\mathrm{K})\end{array}$ & Ref. \\
\hline \multirow{2}{*}{} & $-2.71 \pm 0.09$ & $2.20 \pm 0.22$ & $4.14 \pm 0.16$ & $2552 \pm 188$ & $(a)$ \\
\hline & $-2.83_{-0.36}^{+0.38}$ & $1.97_{-0.52}^{+0.87}$ & $\leq 4.11$ & $2547_{-136}^{+94}$ & $(b)$ \\
\hline & $-2.79 \pm 0.15$ & $2.00 \pm 0.23$ & $4.13 \pm 0.15$ & $2391 \pm 249$ & $(a)$ \\
\hline
\end{tabular}

References. ${ }^{(a)}$ Filippazzo et al. (2015), ${ }^{(b)}$ this work with the adopted values (see Tables C.1-C.2).

predictions from solar metallicity SMHCQ8 (Saumon \& Marley 2008) and DMESTAR (Feiden \& Chaboyer 2012, 2013) isochrones, Filippazzo et al. (2015) derived the predicted radii and masses, and therefore the object effective temperatures using the StefanBoltzmann law as well as the surface gravities. The results are reported in Table 4, and roughly agree within the error bars with those obtained by the ForMoSA forward modeling of the X-shooter spectra of TWA 26 and TWA 29. As the three UpperSco companions HIP 78530 B, HIP 77900 B, and USco 16101913 B scan a similar range of temperatures and surface gravities as TWA 26 and TWA 29, this test appears to support the use of ForMoSA to explore their physical properties. These three companions have no known MIR photometry or spectroscopy, and therefore a direct determination of the bolometric luminosity as done by Filippazzo et al. (2015) is not possible here. Based on a fit using the optic wavelength range, we derive a $v \sin (i)=66 \pm$ $2 \mathrm{~km} \mathrm{~s}^{-1}$ for TWA 29. This is the only object for which we are not limited by the resolving power of the instrument.

USco 1610-1913B. For their study of this companion, Lachapelle et al. (2015) used an incorrect value of $A_{\mathrm{v}}=1.1$ given by Carpenter et al. (2009). Using the method described in Carpenter et al. $(2008,2009)$ derived this relatively high $A_{\mathrm{v}}$ using the optical (Johnson $B$ and $V$, Tycho $B_{\mathrm{T}}$ and $V_{\mathrm{T}}$ ) and NIR (2MASS $J, H$, and $K_{s}$ ) photometry and colors, but an incorrect spectral type (K7) for USco 1610-1913. As described in Appendix A, an updated analysis of the full spectral energy distribution (SED) indicates a later spectral type M0, leading to an extinction value of $A_{\mathrm{v}}=0.09 \pm 0.01 \mathrm{mag}$, which is more consistent with the value from Lallement et al. (2019): $A_{\mathrm{v}}=0.13$. Using $A_{\mathrm{v}}=0.13$ with ForMoSA, we derive an effective temperature of $T_{\text {eff }}=2542_{-104}^{+68} \mathrm{~K}$ and a surface-gravity of $\log (g) \leq$ 4.17 dex, both compatible with a young M9 brown dwarf of very low-gravity for USco 1610-1913 B. However, we find clear nonphysical solutions for the radius and the luminosity (for a young late-M dwarf), which confirms the strong over-luminosity of this source, as already pointed out in Sect. 4.1 and discussed further in Sect. 6. We notice a difference between the RV found from ForMoSA and the one found from the cross-correlation algorithm.

HIP $78530 B$. The extinction maps of Lallement et al. (2019) give a value of $A_{\mathrm{v}}=0.075$ along the line of sight of HIP 78530, somewhat different from the value of $A_{\mathrm{v}}=0.5$ used by Lachapelle et al. (2015) and from Carpenter et al. (2009). We adopted the former value in our analysis. ForMoSA finds $T_{\text {eff }}=2679_{-119}^{+118} \mathrm{~K}$, in agreement with the M8 spectral type of the source (Luhman et al. 2003; Filippazzo et al. 2015, this work). The analysis of the continuum-subtracted spectrum yields a surface-gravity estimate compatible with the intermediategravity class of the object (Sect. 4.1). The luminosity value of $\log \left(L / L_{\odot}\right)=-2.87_{-0.15}^{+0.15}$ is also compatible with expected $\log \left(L / L_{\odot}\right)=-2.5$ to -3.0 found by Filippazzo et al. (2015) for young M8 $\beta$ and M8 $\gamma$ BDs. Finally, the effective temperature $T_{\text {eff }}$ and surface-gravity are consistent with the values from Lafrenière et al. (2011).

HIP $77900 B$. With an extinction value of $A_{\mathrm{v}}=0.07$ (Lallement et al. 2019), the resulting effective temperature of $T_{\text {eff }}=2602_{-97}^{+117} \mathrm{~K}$ given by ForMoSA is consistent with the later M9 \pm 0.5 spectral type empirically derived for this companion. The estimated surface-gravity of $\log (g) \leq 4.36 \mathrm{dex}$ is consistent with the intermediate-gravity classification of Allers \& Liu (2013), and the luminosity value of $\log \left(L / L_{\odot}\right)=-2.89_{-0.13}^{+0.15}$ is compatible with the values found for young M9 $\beta \gamma$ brown dwarfs (Filippazzo et al. 2015).

\section{Emission line properties}

Our new high-S/N medium-resolution optical spectra allow us to identify the faint $\mathrm{H}_{\alpha}(656.28 \mathrm{~nm})$ emission lines of the three companions and of four out of the six free-floating objects of our sample. Figure 14 shows the line profiles at each epoch. We checked for the emission line detection in the traces to ensure it was not produced by an uncorrected bad pixel (see Sect. 3). $\mathrm{H}_{\alpha}$ lines are commonly attributed to magnetospheric accretion (Manara et al. 2013, 2015; Natta et al. 2004) or chromospheric activity (e.g., White \& Basri 2003). The three objects (USco 1610-1913 B, USco 1606-2335, and USco 16082315) showing the strongest $\mathrm{H}_{\alpha}$ lines also display other Balmer lines, $\mathrm{H}_{\beta}(486.1 \mathrm{~nm})$ and $\mathrm{H}_{\gamma}(434.0 \mathrm{~nm})$, in their spectra. We tentatively identify Ca II-H $(393.4 \mathrm{~nm})$ and Ca II-K $(396.9 \mathrm{~nm})$ emission lines in the spectrum of USco 1610-1913 B as well. The Ca II lines can also be related to accretion or activity (Herczeg \& Hillenbrand 2008). We report the apparent fluxes of these emission lines and $10 \%$ width of the $\mathrm{H}_{\alpha}$ line in Table C.3. The noisy spectral continua of our objects prevented us from obtaining a robust determination of the continuum and the equivalent widths. Both origins (accretion and activity) for our targets are investigated below. 


\subsection{Accretion rate determination}

The accretion rates of each object are reported in Table C.5. They were derived following the relation of Alcalá et al. (2017):

$\dot{M}_{\mathrm{acc}}=\left(1-\frac{R_{\mathrm{obj}}}{R_{\mathrm{in}}}\right)^{-1} \frac{L_{\mathrm{acc}} R_{\mathrm{obj}}}{G M_{\mathrm{obj}}} \approx 1.25 \frac{L_{\mathrm{acc}} R_{\mathrm{obj}}}{G M_{\mathrm{obj}}}$,

where $L_{\text {acc }}$ is the accretion luminosity, $R_{\text {in }}$ is the inner disk radius, $R_{\mathrm{obj}}$ and $M_{\mathrm{obj}}$ are the radius and mass of the objects, respectively. This is assuming $R_{\mathrm{obj}} / R_{\text {in }}=0.2$ (Alcalá et al. 2017) which holds in our mass range, irrespective of the object configuration (companion or isolated). The accretion luminosities were estimated beforehand from the line fluxes of the Ca II lines and the Balmer lines using the relationships given in Rigliaco et al. (2012) and Alcalá et al. (2017), respectively. Radii and masses were in addition inferred from the evolutionary model predictions as indicated in Table 5.

Alternatively, we derived accretion rates based on $10 \% \mathrm{H}_{\alpha}$ width following the relations of Natta et al. (2004):

$\log \left(\dot{M}_{\mathrm{acc}}\right)=-12.89( \pm 0.3)+9.7( \pm 0.7) \times 10^{-3} \mathrm{H}_{\alpha} 10 \%$.

The $10 \% \mathrm{H}_{\alpha}$ accretion rates are also reported in Table C.5. They tend to be higher than the ones inferred from the Balmer and $\mathrm{Ca}$ II line fluxes. A similar divergence between the different accretion proxies is found for the young companion SR 12C (Santamaría-Miranda et al. 2018). The accretion rates of USco 1610-1913 B are compatible with those of the 1-5 Myr late-M sub-stellar companions FW Taub (Bowler et al. 2014), DH Tau b (Zhou et al. 2014), and GQLup b (Wu et al. 2017b), which had the lowest recorded accretion rates among known accreting companions. The accretion rates of HIP $78530 \mathrm{~B}$ and HIP $77900 \mathrm{~B}$ are found to be an order of magnitude lower, indicating that these objects are nonaccretors.

\subsection{Chromospheric activity}

The weakness of the lines suggests the emission arises mostly from chromospheric activity. Manara et al. (2013) provides the $L_{\text {acc,noise }} / L_{\text {bol }}$ values expected from chromospheric activity for young stars and BDs down to the M9 spectral type:

$\log \left(L_{\text {acc,noise }} / L_{\text {bol }}\right)=(6.17 \pm 0.53) \times \log \left(T_{\text {eff }}\right)-(24.54 \pm 1.88)$

All of our objects apart from USco 1608-2315 have $\log \left(L_{\mathrm{acc}} / L_{\mathrm{bol}}\right)$ values below or comparable to the activity threshold $\log \left(L_{\mathrm{acc}, \text { noise }} / L_{\mathrm{bol}}\right)$ corresponding to their $T_{\mathrm{eff}}$ (estimated from ForMoSA), indicating that the lines are indeed likely related to chromospheric activity. Herczeg et al. (2009) also identify a $\mathrm{H}_{\alpha}$ line on USco 1607-2211 of similar intensity and came to the same conclusion. We note that this criterion applied to the $\mathrm{H}_{\alpha}$ line of GQ Lup b as observed in April 2016 (Wu et al. 2017b) indicates that the line mostly arises from chromospheric activity in spite of accretion at this epoch ${ }^{5}$. The $10 \%$ width of the $\mathrm{H}_{\alpha}$ line may also discriminate between the accretion (large values) and chromospheric activity (small values). A threshold of $200 \mathrm{~km} \mathrm{~s}^{-1}$ is generally adopted for BDs (Jayawardhana et al. 2003). All of our objects but USco 1608-2315 have a $10 \% \mathrm{H}_{\alpha}$ below the

5 We considered the $T_{\text {eff }}$ from Seifahrt et al. (2007) and Zhou et al. (2014) and the luminosity from Zhou et al. (2014) to compute the $\log \left(L_{\mathrm{acc}, \text { noise }} / L_{\mathrm{bol}}\right)=-3.8$ to -3.48 value and compare it to $\log \left(L_{\mathrm{acc}} / L_{\mathrm{bol}}\right)=-3.9$ to -2.9 of the object.

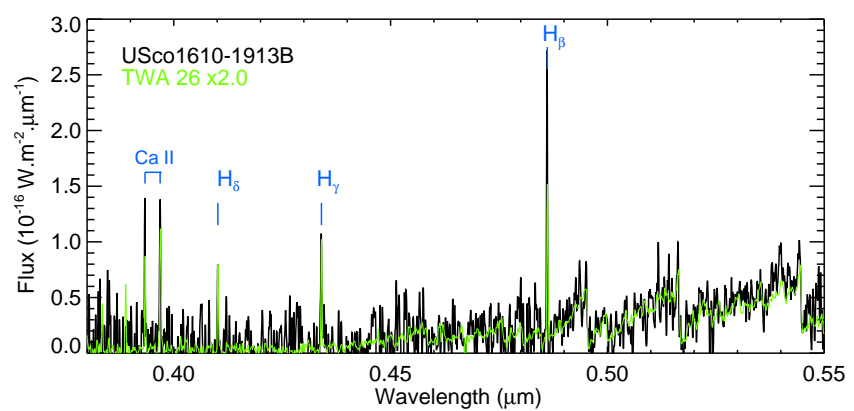

Fig. 13. Comparison of the UVB spectrum of USco 1610-1913 B (black) and TWA 26 (green) re-normalized to match the spectral continuum of the companion. Emission lines identified in the spectra are reported.

$200 \mathrm{~km} \mathrm{~s}^{-1}$ limit, supporting line production by chromospheric activity. Therefore, USco $1608-2315$ is likely the only true accreting object in our sample. The source also has a tentative disk excess (Sect. 2). Thus, we add USco 1608-2315 to the short list of free-floating accretors with masses below $30 M_{\text {Jup }}$ (Herczeg et al. 2009; Bayo et al. 2012; Joergens et al. 2013; Alcalá et al. 2014; Boucher et al. 2016; Lodieu et al. 2018).

To further support these conclusions for a few specific objects, Fig. 13 compares the UVB spectra of USco 1610-1913 B and TWA 26 scaled to the distance of USco 1610-1913 B. The flux from TWA 26 had to be multiplied by an additional factor of two to match the flux level of USco 1610-1913 B (see Sect. 6.2). The pseudo-continuum of USco 1610-1913 B is well reproduced by a template with emission lines due to chromospheric activity (Manara et al. 2013). The $\mathrm{H}_{\gamma}$ and $\mathrm{H}_{\delta}$ lines have similar strengths but the $\mathrm{H}_{\beta}$ line appears more luminous in the companion spectrum.

Figure 14 shows that the two free-floating objects with the strongest lines (USco 1608-2315 and USco 1606-2335) display significant short-term variability. The lines are not detected in the optical spectra of these objects obtained in May 2007 (Lodieu et al. 2011). The $\mathrm{H}_{\alpha}$ lines of USco 1607-2211 and USco 1610-2239 do not show obvious variability in our data, but are not detected in May 2007 (Lodieu et al. 2011) either. It is possible that the nondetections from 2007 arise from a degraded sensitivity of the low-resolution data from Lodieu et al. (2011). Active M-dwarfs are known to display such variability from chromospheric activity (e.g., Gizis et al. 2002), which strengthens our conclusions on the line origins. The variability of USco 1608-2315 could also stem from variation of the accretion rate (Aguayo et al., in prep.; Bonnefoy et al., in prep.).

\section{Discussion}

\subsection{Revisiting the physical association of HIP $77900 B$ and USco 1610-1913 B with Gaia}

HIP 77900 B and USco 1610-1913 B are sufficiently bright and distant from their primary for them to have reported Gaia parallaxes. This is to our knowledge the first case for which the physical association of young imaged BD companions with their primary stars can be investigated based on the individual five-parameters astrometric solutions of the system components.

\subsubsection{The case of HIP 77900 B}

The Gaia-DR2 parallax of HIP 77900 A confirms that this system would be extreme if bound $\left(22.3^{\prime \prime}=3375\right.$ au projected separation). Very wide systems such as HIP 77900 are expected 

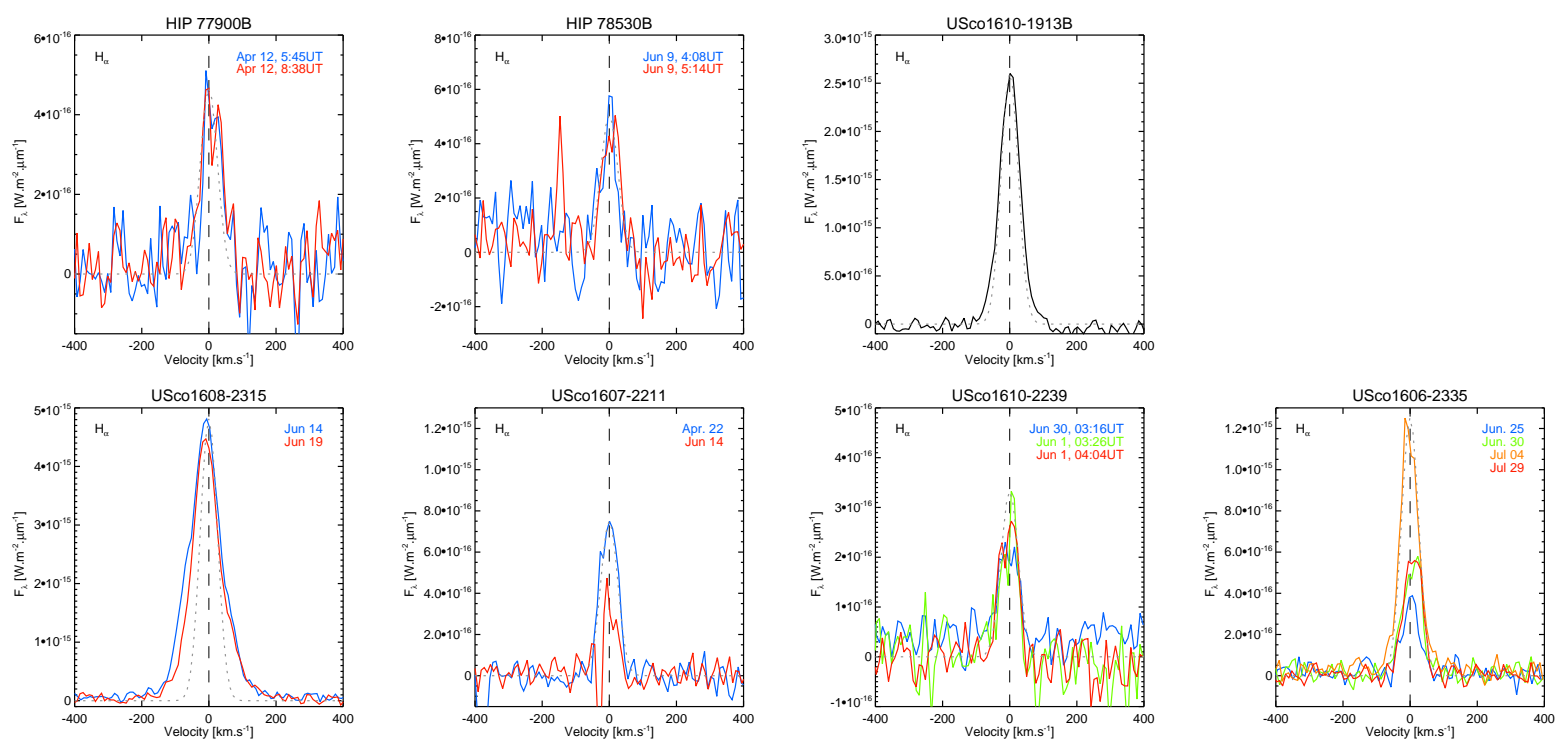

Fig. 14. $\mathrm{H}_{\alpha}$ line profiles for six of the targets with the strongest line emission. The dotted gray line corresponds to an estimate of the line spread function.

to be rare (e.g., Baron et al. 2018). The companion has not been observed at multiple epochs so it could in principle be a background star. However, the close resemblance of the spectrum of the companion to those of free-floating analogs from our library of Upper-Sco objects identifies it as a likely member of this association. Therefore, HIP 77900 A and B are either coeval or aligned by chance within the association. Their respective distances $\left(191_{+43}^{-30} \mathrm{pc}\right.$ for $\mathrm{A}$, and $151.4_{-2.7}^{+2.8} \mathrm{pc}$ for $\left.\mathrm{B}\right)$ reveal a $1.4 \sigma$ difference. There is in addition a $1.8 \sigma$ difference between the proper motion in declination of the two objects. The $p$-value on the difference on the three astrometric parameters of A and B (0.06) does not allow us to firmly conclude whether or not the system is coeval. The Re-normalized Unit Weight Error (RUWE) values of A and B (0.97 and 1.21) indicate robust solutions. The galactic Cartesian coordinates of HIP $77900 \mathrm{~A}\left(X_{\mathrm{A}}=137.8 \pm 2.6 \mathrm{pc}, Y_{\mathrm{A}}=-35.5 \pm 0.7 \mathrm{pc}\right.$, $Z_{\mathrm{A}}=51.8 \pm 1.0 \mathrm{pc}$ ) computed following Gagné et al. (2014) are well within the range of expected values for Upper-Sco (Galli et al. 2018), contrary to those of $\mathrm{B}\left(X_{\mathrm{B}}=173.8_{-27.3}^{+39.1} \mathrm{pc}\right.$, $\left.Y_{\mathrm{B}}=-44.7_{-10.1}^{+7.1} \mathrm{pc}, Z_{\mathrm{B}}=65.4_{-10.3}^{+14.8} \mathrm{pc}\right)$ which is at odds with the youth of the object. However, we find photometric distances of $151_{-24}^{+35} \mathrm{pc}$ and $150_{+22}^{-17} \mathrm{pc}$ for HIP $77900 \mathrm{~B}$ using the fluxcalibrated spectra of the spectrally analogous M8.5 dwarfs from our sample (USco 1607-2211 and USco 1610-2239, respectively) with Gaia parallaxes. These photometric distances are more consistent with the Gaia distance of A and would strengthen the case of a coeval system. However, we found a RV of $19.3 \pm 1.2 \mathrm{~km} \mathrm{~s}^{-1}$ for the $\mathrm{B}$ component which is not consistent with the $1.3 \pm$ $2.6 \mathrm{~km} \mathrm{~s}^{-1}$ found by Gontcharov (2006) for HIP $77900 \mathrm{~A}$. This result raises severe doubts about the gravitational link between the two objects, and the next Gaia data releases of the relative astrometry of A and B will be needed to firmly conclude on their physical association.

\subsubsection{The case of USco 1610-1913 B}

The spectral properties and measured distances of USco 16101913 B clearly confirm its membership to Upper-Sco. USco 1610$1913 \mathrm{~B}$ and A have been shown to have a common proper motion from 2007 to 2012 and have been proposed to be bound
(Kraus et al. 2014, and ref therein). However, the recent DR2 solutions of these two objects diverge in proper motion (2.0 and $2.4 \sigma$ significance in $\mu_{\alpha}$ and $\mu_{\delta} \cdot \cos (\delta)$, respectively) and in distance $(1.4 \sigma ; \mathrm{B}$ is $10.3 \mathrm{pc}$ closer than $\mathrm{A})$. A $\chi^{2}$ test on the difference on the three astrometric parameters of each system component (parallaxes and proper motions) taking into account the correlations gives a $p$-value of 0.01 which is in favor of a physical bond between the two objects. The p-value accounts for the slight underestimation of the errors (Lindegren et al. 2018). The reliability of the Gaia astrometry may however be questioned for this particular system. Indeed, the $\sim 0.145^{\prime \prime}$ M-dwarf companion to USco 1610-1913 A (Kraus et al. 2008) is unresolved in the DR2. The RUWE index is proposed as a more reliable and informative goodness-of-fit statistic than for instance the astrometric excess noise (Lindegren et al. 2018, Gaia technical note Gaia-C3-TN-LU-LL-124-01). The RUWE of USco 1610-1913 A (1.63) is above the threshold of 1.4 and indicates that the observations are inconsistent with a simple five-parameter astrometric model while B (RUWE $=1.19)$ shows a more reliable solution. The next releases of Gaia will here again solve this ambiguity.

If we keep the hypothesis that the system is bound (or even coeval) and located at the distance of the B component, USco 1610-1913 B remains 4.5 times more luminous than free-floating analogs from the Upper-Sco association. This is illustrated in Fig. 5 using USco 1607-2211 and USco 1610-2239 for comparison, which both have high-quality astrometric solutions. In addition, the probability of a chance alignment within the association is small. Aller et al. (2013) identified USco 16101913 B while searching for distant companions within 30 arcseconds of Upper-Sco stars and with optical (Pan-Starrs) and NIR (UKIDSS) colors compatible with young, cool objects. Using the now-available Pan-Starrs data (Chambers et al. 2016), we confirm that USco 1610-1913 B belongs to one of the three objects with the reddest $i-z$ and $i-y$ colors within 5 arcmin of USco 1610-1913 A. It is also the only object in this field with colors typical of M7-M9 Upper-Sco objects. Dahm et al. (2012) determined a RV of $-6.91 \pm 0.27 \mathrm{~km} \mathrm{~s}^{-1}$ for USco 16101913 A. USco 1610-1913 B is the unique target for which we find a strong discrepancy between the RV determined by ForMoSA 
Table 5. Comparison between parameters from ForMoSA and parameters in using evolutionary models (Chabrier et al. 2000) (BT-SETTL15/ForMoSA independent).

\begin{tabular}{|c|c|c|c|c|c|c|c|c|c|}
\hline \multirow[b]{2}{*}{ Object } & \multicolumn{4}{|c|}{ ForMoSA } & \multicolumn{5}{|c|}{ Bolometric correction + Evolutionary models } \\
\hline & $\log \left(L / L_{\odot}\right)$ & $\begin{array}{c}R \\
\left(R_{\text {Jup }}\right)\end{array}$ & $\begin{array}{c}\log (g) \\
(\mathrm{dex})\end{array}$ & $\begin{array}{l}T_{\text {eff }} \\
(\mathrm{K})\end{array}$ & $\log \left(L / L_{\odot}\right)$ & $\begin{array}{c}R \\
\left(R_{\text {Jup }}\right)\end{array}$ & $\begin{array}{c}\log (g) \\
(\operatorname{dex})\end{array}$ & $\begin{array}{l}T_{\text {eff }} \\
(\mathrm{K})\end{array}$ & $\begin{array}{c}\mathrm{M} \\
\left(M_{\text {Jup }}\right)\end{array}$ \\
\hline Sco 1610-1913 B ${ }^{(1)}$ & $-2.25_{-0.10}^{+0.10}$ & $3.87_{-0.12}^{+0.24}$ & $\leq 4.17$ & $2542_{-104}^{+68}$ & $2.13 \pm 0.17$ & $6 \pm 0.7$ & $4.03 \pm 0.20$ & $2827 \pm 169$ & $\overline{57 \pm 28}$ \\
\hline HIP $77900 B^{(1)}$ & $-2.89_{-0.13}^{+0.15}$ & $1.76_{-0.12}^{+0.15}$ & $\leq 4.36$ & $2602_{-97}^{+117}$ & $-2.59 \pm 0.16$ & $2.8 \pm 0.4$ & $4.02 \pm 0.17$ & $2611 \pm 185$ & $34 \pm 14$ \\
\hline HIP $78530 \mathrm{~B}^{(1)}$ & $-2.87_{-0.15}^{+0.15}$ & $1.83_{-0.14}^{+0.16}$ & $\leq 4.34$ & $\begin{array}{r}2679_{-119}^{+118} \\
\end{array}$ & $-2.68 \pm 0.17$ & $2.6 \pm 0.4$ & $4.00 \pm 0.13$ & $2544 \pm 179$ & $28 \pm 10$ \\
\hline US & $-3.41_{-0.18}^{+0.18}$ & $1.13_{-0.08}^{+0.13}$ & $\leq 4$ & $2403_{-152}^{+122}$ & & $9 \pm 0.2$ & $3.99 \pm 0.09$ & $2044 \pm 203$ & $\overline{14 \pm 4}$ \\
\hline USco 1608-2232 & $-3.09_{-0.14}^{+0.15}$ & $1.63_{-0.12}^{+0.19}$ & $\leq 4.09$ & $2409_{-99}^{+81}$ & $-2.96 \pm 0.20$ & $2.1 \pm 0.3$ & $4.01 \pm 0.06$ & $2262 \pm 182$ & $17 \pm 4$ \\
\hline USco 1606-2335 & $-3.11_{-0.16}^{+0.16}$ & $1.46_{-0.10}^{+0.15}$ & $\leq 4.36$ & $2519_{-141}^{+114}$ & $-3.05 \pm 0.20$ & $2.1 \pm 0.2$ & $4.01 \pm 0.06$ & $2215 \pm 179$ & $16 \pm 4$ \\
\hline USco 1610-2239 & $-2.88_{-0.14}^{+0.17}$ & $1.93_{-0.14}^{+0.24}$ & $\leq 4.01$ & $2499_{-108}^{+102}$ & $-2.75 \pm 0.20$ & $2.5 \pm 0.3$ & $3.97 \pm 0.08$ & $2467 \pm 173$ & $24 \pm 7$ \\
\hline USco 1608-2315 & $-2.86_{-0.15}^{+0.11}$ & $2.00_{-0.09}^{+0.12}$ & $\leq 4.16$ & $2487_{-147}^{+81}$ & $-2.71 \pm 0.20$ & $2.5 \pm 0.4$ & $3.98 \pm 0.08$ & $2474 \pm 179$ & $24 \pm 8$ \\
\hline USco 1607-2211 & $-2.84_{-0.11}^{+0.07}$ & $1.92_{-0.06}^{+0.05}$ & $\leq 4.05$ & $2557_{-117}^{+65}$ & $-2.76 \pm 0.24$ & $2.5 \pm 0.4$ & $3.98 \pm 0.09$ & $2461 \pm 204$ & $24 \pm 8$ \\
\hline
\end{tabular}

Notes. The luminosity has been determined using the bollometric corrections at $J$ and $K$ bands from Filippazzo et al. (2015). For the luminosity of our reference objects we directly use the Filippazzo et al. (2015) values. We also provide the mass values. ${ }^{(1)}$ With the hypothesis that companions are at the same distance as the primary star.

and the cross-correlation approach $\left(11.5 \pm 0.4\right.$ and $0.9 \mathrm{~km} \mathrm{~s}^{-1}$ respectively). Both values are in addition inconsistent with the RV derived by Dahm et al. (2012) for the A component, and are therefore inconclusive. Although we cannot firmly exclude that USco 1610-1913 A and B are simply members of UpperSco aligned by chance, parallaxes and common proper motion do support possibility that they form at least a comoving and possible coeval pair.

\subsection{The over-luminosity of USco 1610-1913 B}

Evolutionary models of Chabrier et al. (2000) predict a $T_{\text {eff }}=2827 \pm 169 \mathrm{~K}$ for USco 1610-1913 B using the bolometric luminosity of the source. This corresponds to a M5 brown dwarf (see Table 5), incompatible with the spectral type of M9 \pm 0.5 derived from our empirical analysis. Our results using the ForMoSA code (see Sect. 4.3) further confirm this overluminosity based on model atmosphere fits. There are several other sources in the literature which are similarly overluminous, such as USco 1602-2401 B (Aller et al. 2013) or 2MASS J162243.85-195 105.7 (Dahm et al. 2012). Aller et al. (2013) proposed that differences in the accretion history of USco 1610-1913 B could play a role in such a discrepancy as proposed by Baraffe et al. (2012). For late K- and M-dwarfs, chromospheric activity as found in our spectra decreases the objects $T_{\text {eff }}$ and increases their radii (López-Morales 2007; Morales et al. 2008). Based on a sample of $669 M<1 M_{\odot}$ nonaccreting stars from the Palomar/Michigan State University catalog (PMSU; Reid et al. 1995; Hawley et al. 1996), Stassun et al. (2012) proposed empirical relations to determine the bias induced by chromospheric activity:

$$
\frac{\Delta T_{\mathrm{eff}}}{T_{\mathrm{eff}}}(\%)=(-3.12 \pm 3.15) \times\left(\log \left(\frac{L_{\mathrm{H}_{\alpha}}}{L_{\mathrm{bol}}}\right)+4\right)+(-5.1 \pm 0.7)^{\prime}
$$

$\frac{\Delta R}{R}(\%)=(8.00 \pm 7.63) \times\left(\log \left(\frac{L_{\mathrm{H}_{\alpha}}}{L_{\mathrm{bol}}}\right)+4\right)+(11.2 \pm 1.6)$
Despite these variations of the radius and $T_{\text {eff }}$ of the atmosphere, the bolometric luminosity remains constant. This effect cannot explain the over-luminosity of USco 1610-1913 B. An alternative explanation would be that the companion is an unresolved quadruple system presumably with all components having similar spectral types in this case. Such systems are expected to be rare among A- and FG-type stars (Raghavan et al. 2010; De Rosa et al. 2014; Tokovinin 2014). The system would be even more exotic and rare considering that USco 1610-1913 A is a binary and that USco 1610-1913 B has to be composed of four identical objects to account for its overluminosity. Quadruples tend to be found as $2+2$ tight binary systems. The discovery of a quadruple system made of two pairs of M5 eclipsing binaries in Upper-Sco (Wang et al. 2018) shows that such objects exist in isolation. To our knowledge, adaptive-optics images of the system have not displayed PSF-elongation or resolved the companion as a higher-order object but new NIR high-resolution $(R 80000$ to 100 000) spectrographs (ESO/NIRPS, CRIRES+) could investigate the multiplicity of USco 1610-1913B in the near future.

\section{Conclusion}

Based on medium-resolution spectra obtained with the $\mathrm{X}$-shooter spectrograph at the VLT, we carried out an indepth characterization of three low-mass BD companions on wide-orbits, namely USco 161031.9-16191305 B, HIP 77900 B, and HIP $78530 \mathrm{~B}$ of the Upper-Scorpius association, together with six young isolated brown dwarfs of similar spectral types and ages. The X-shooter data yield the first medium-resolution optical spectra of the companion objects. We can summarize the main results as follows:

1. The re-investigation of the spectral classification of the three companions USco 1610-1913 B, HIP 77900 B and HIP $78530 \mathrm{~B}$ confirms that they have spectral types M9 \pm 0.5 , M9 \pm 0.5 , and M8 \pm 0.5 , respectively. HIP $77900 \mathrm{~B}$ and HIP $78530 \mathrm{~B}$ are identified as young, intermediatesurface-gravity brown dwarfs, whereas USco 1610-1913 B is confirmed as a very-low-surface-gravity brown dwarf. 
2. The development and use of the ForMoSA forward modeling code relying on the Nested Sampling procedure enables us to infer posterior probability distributions of the physical properties $\left(T_{\mathrm{eff}}, \log (g), R, L\right.$ and extinction) of USco 1610-1913 B, HIP 77900 B and HIP 78530 B using the BT-SETTL15 atmospheric models and the X-shooter spectra. We find that generally, the models fail to reproduce the pseudo-continuum of the X-shooter spectra over a broad range of wavelengths. Our solutions are mainly affected by the choice of the spectral range considered to estimate the best fit, and the fitting error bars remain about one to two orders of magnitude smaller given the high $\mathrm{S} / \mathrm{N}$ of the $\mathrm{X}$-shooter spectra. Finally, when the extinction is considered as a free parameter, the ForMoSA fitting solutions are considerably improved at all wavelengths with extinction values of 1.6-2.6 mag suggesting a clear deficiency in the dust grain modeling of the BT-SETTL15 atmospheric models. These inconsistencies currently limit our ability to investigate the chemical abundance of heavy elements in the atmosphere of these young brown dwarfs, which could trace different formation mechanisms. Finally, the key physical properties such as $T_{\text {eff }}, \log (g)$, and $L$ are in agreement with the empirical analysis, but indicate a clear over-luminosity for USco 1610-1913 B.

3. The study of the medium-resolution optical part of the $\mathrm{X}$-shooter spectra allowed us to identify the presence of various Balmer lines for the three companions USco 16101913 B, HIP $77900 \mathrm{~B}$, and HIP $78530 \mathrm{~B}$, and the two isolated brown dwarfs USco 1608-2315 and USco 1607-2211, and allowed us to investigate their origin. Their low accretion rate, low accretion luminosity and low $10 \%$ width tend to promote them as signatures tracing chromospheric activity except for USco 1608-2315, which adds to the limited population of accreting free-floating young brown dwarfs with masses below $30 M_{\text {Jup }}$.

4. The nature of USco 1610-1913 B and HIP 77900 B was revisited and discussed in the context of the new Gaia DR2 results. The Gaia solutions of USco 1610-1913 A are probably affected by the binarity of USco 1610-1913 A itself. For HIP $77900 \mathrm{~B}$, the parallax is surprisingly inconsistent with the photometric distance. Nevertheless, a coeval (bound or unbound) configuration remains the most plausible one for both systems. Finally, we showed that the over-luminosity of USco 1610-1913 B cannot be explained by chromospheric activity suggesting that it might be a high-order multiple component if we want to reconcile spectral type and observed luminosity. Further studies using high-resolution spectrographs $\left(R_{\lambda} \simeq 80000-100000\right)$ like ESO3.6 m/NIRPS or VLT/CRIRES+ are required to explore such a hypothesis.

Acknowledgements. The authors thank the ESO staff for conducting the observations. We are grateful to D. Lachapelle for providing the spectra of HIP $78530 \mathrm{~B}$ and USco 1610-1913 B. We also thank J. Bouvier for advising us on accretion process and chromospheric activity effects. We acknowledge financial support from the Programme National de Planétologie (PNP), the Programme National de Physique Stellaire (PNPS) of CNRS-INSU in France, and the Agence Nationale de la Recherche (GIPSE project; grant ANR-14-CE33-0018). This research has made use of the SIMBAD database and VizieR catalogue access tool (operated at CDS, Strasbourg, France). This research has made use of NASA's Astrophysics Data System and of the Extrasolar Planet Encyclopedia (http:// exoplanet.eu/). This publication makes use of VOSA, developed under the Spanish Virtual Observatory project supported from the Spanish MINECO through grant AyA2017-84089. G-DM acknowledges the support of the DFG priority program SPP 1992 "Exploring the Diversity of Extrasolar Planets" (KU 2849/7-1).

\section{References}

Alam, S., Albareti, F. D., Allende Prieto, C., et al. 2015, ApJS, 219, 12 Alcalá, J. M., Natta, A., Manara, C. F., et al. 2014, A\&A, 561, A2

Alcalá, J. M., Manara, C. F., Natta, A., et al. 2017, A\&A, 600, A20

Allard, F., Hauschildt, P. H., Alexander, D. R., Tamanai, A., \& Schweitzer, A 2001, ApJ, 556, 357

Allard, F., Homeier, D., \& Freytag, B. 2011, ASP Conf. Ser., 448, 91

Allard, F., Homeier, D., \& Freytag, B. 2012, Phil. Trans. R. Soc. London, Ser. A, 370,2765

Allard, F., Homeier, D., Freytag, B., et al. 2013, Mem. Soc. Astron. It. Suppl., 24,128

Allers, K. N. 2012, IAU Symp., 282, 105

Allers, K. N., \& Liu, M. C. 2013, ApJ, 772, 79

Allers, K. N., Jaffe, D. T., Luhman, K. L., et al. 2007, ApJ, 657, 511

Aller, K. M., Kraus, A. L., Liu, M. C., et al. 2013, ApJ, 773, 63

Ardila, D., Martín, E., \& Basri, G. 2000, AJ, 120, 479

Baraffe, I., Chabrier, G., Barman, T. S., Allard, F., \& Hauschildt, P. H. 2003, A\&A, 402, 701

Baraffe, I., Vorobyov, E., \& Chabrier, G. 2012, ApJ, 756, 118

Baraffe, I., Homeier, D., Allard, F., \& Chabrier, G. 2015, A\&A, 577, A42

Barman, T. S., Macintosh, B., Konopacky, Q. M., \& Marois, C. 2011, ApJ, 735, L39

Baron, F., Artigau, É., Rameau, J., et al. 2018, AJ, 156, 137

Bate, M. R., Bonnell, I. A., \& Bromm, V. 2002, MNRAS, 332, L65

Bayo, A., Rodrigo, C., Barrado, Y., Navascués, D., et al. 2008, A\&A, 492, 277

Bayo, A., Barrado, D., Huélamo, N., et al. 2012, A\&A, 547, A80

Bayo, A., Barrado, D., Allard, F., et al. 2017, MNRAS, 465, 760

Béjar, V. J. S., Zapatero Osorio, M. R., Pérez-Garrido, A., et al. 2008, ApJ, 673, L185

Biller, B., Allers, K., Liu, M., Close, L. M., \& Dupuy, T. 2011, ApJ, 730, 39

Bonnefoy, M., Chauvin, G., Lagrange, A. M., et al. 2014, A\&A, 562, A127

Bonnefoy, M., Perraut, K., Lagrange, A.-M., et al. 2018, A\&A, 618, A63

Boucher, A., Lafrenière, D., Gagné, J., et al. 2016, ApJ, 832, 50

Bowler, B. P., Liu, M. C., Kraus, A. L., Mann, A. W., \& Ireland, M. J. 2011, ApJ, 743, 148

Bowler, B. P., Liu, M. C., Kraus, A. L., \& Mann, A. W. 2014, ApJ, 784, 65

Bowler, B. P., Andrews, S. M., Kraus, A. L., et al. 2015, ApJ, 805, L17

Bubar, E. J., Schaeuble, M., King, J. R., Mamajek, E. E., \& Stauffer, J. R. 2011 AJ, 142, 180

Burrows, A., Marley, M., Hubbard, W. B., et al. 1997, ApJ, 491, 856

Carpenter, J. M., Mamajek, E. E., Hillenbrand, L. A., \& Meyer, M. R. 2006, ApJ, 651, L49

Carpenter, J. M., Bouwman, J., Silverstone, M. D., et al. 2008, ApJS, 179, 423

Carpenter, J. M., Mamajek, E. E., Hillenbrand, L. A., \& Meyer, M. R. 2009, ApJ, 705,1646

Chabrier, G., Baraffe, I., Allard, F., \& Hauschildt, P. 2000, ApJ, 542, L119

Chambers, K. C., Magnier, E. A., Metcalfe, N., et al. 2016, ArXiv e-prints [arXiv:1612.05560]

Charnay, B., Bézard, B., Baudino, J. L., et al. 2018, ApJ, 854, 172

Cruz, K. L., Reid, I. N., Liebert, J., Kirkpatrick, J. D., \& Lowrance, P. J. 2003, AJ, 126, 2421

Cruz, K. L., Reid, I. N., Kirkpatrick, J. D., et al. 2007, AJ, 133, 439

Cushing, M. C., Rayner, J. T., \& Vacca, W. D. 2005, ApJ, 623, 1115

Cushing, M. C., Marley, M. S., Saumon, D., et al. 2008, ApJ, 678, 1372

Cutri, R. M., Skrutskie, M. F., van Dyk, S., et al. 2003, 2MASS All Sky Catalog of Point Sources (Washington: NASA/IPAC)

Cutri, R. M., et al. 2013, VizieR Online Data Catalog: II/328

Dahm, S. E., Slesnick, C. L., \& White, R. J. 2012, ApJ, 745, 56

Dai, Y., Wilner, D. J., Andrews, S. M., \& Ohashi, N. 2010, AJ, 139, 626

David, T. J., Hillenbrand, L. A., Gillen, E., et al. 2019, ApJ, 872, 161

Dawson, P., Scholz, A., Ray, T. P., et al. 2013, MNRAS, 429, 903

de Bruijne, J. H. J. 1999, MNRAS, 310, 585

De Rosa, R. J., Patience, J., Wilson, P. A., et al. 2014, MNRAS, 437, 1216

de Zeeuw, P. T., Hoogerwerf, R., de Bruijne, J. H. J., Brown, A. G. A., \& Blaauw, A. 1999, AJ, 117, 354

Delorme, P., Dupuy, T., Gagné, J., et al. 2017a, A\&A, 602, A82

Delorme, P., Schmidt, T., Bonnefoy, M., et al. 2017b, A\&A, 608, A79

Draine, B. T. 2003a, ARA\&A, 41, 241

Draine, B. T. 2003b, ApJ, 598, 1017

Draine, B. T. 2003c, ApJ, 598, 1026

Esplin, T. L., Luhman, K. L., Miller, E. B., \& Mamajek, E. E. 2018, AJ, 156, 75 Feiden, G. A., \& Chaboyer, B. 2012, ApJ, 761, 30

Feiden, G. A., \& Chaboyer, B. 2013, ApJ, 779, 183

Filippazzo, J. C., Rice, E. L., Faherty, J., et al. 2015, ApJ, 810, 158

Fitzpatrick, E. L., \& Massa, D. 2007, ApJ, 663, 320

Freudling, W., Romaniello, M., Bramich, D. M., et al. 2013, A\&A, 559, A96 
Gaia Collaboration (Brown, A. G. A., et al.) 2018, A\&A, 616, A1 Gagné, J., Lafrenière, D., Doyon, R., Malo, L., \& Artigau, É. 2014, ApJ, 783, 121

Gagné, J., Mamajek, E. E., Malo, L., et al. 2018, ApJ, 856, 23

Galli, P. A. B., Joncour, I., \& Moraux, E. 2018, MNRAS, 477, L50

Garrison, R. F. 1967, ApJ, 147, 1003

Gizis, J. E., Reid, I. N., \& Hawley, S. L. 2002, AJ, 123, 3356

Gontcharov, G. A. 2006, Astron. Lett., 32, 759

Hauschildt, P. H., Baron, E., \& Allard, F. 1997, ApJ, 483, 390

Hawley, S. L., Gizis, J. E., \& Reid, I. N. 1996, AJ, 112, 2799

Helling, C., \& Woitke, P. 2006, A\&A, 455, 325

Henden, A. A., Templeton, M., Terrell, D., et al. 2016, VizieR Online Data Catalog: II/336

Herczeg, G. J., \& Hillenbrand, L. A. 2008, ApJ, 681, 594

Herczeg, G. J., Cruz, K. L., \& Hillenbrand, L. A. 2009, ApJ, 696, 1589

Hester, J. J., Scowen, P. A., Sankrit, R., et al. 1996, AJ, 111, 2349

Houk, N., \& Smith-Moore, M. 1988, Michigan Catalogue of Two-dimensional Spectral Types for the HD Stars (Michigan: University of Michigan), 4

Ireland, M. J., Kraus, A., Martinache, F., Law, N., \& Hillenbrand, L. A. 2011, ApJ, 726, 113

Jayawardhana, R., Ardila, D. R., Stelzer, B., \& Haisch, K. E., Jr. 2003, AJ, 126, 1515

Joergens, V., Bonnefoy, M., Liu, Y., et al. 2013, A\&A, 558, L7

Kausch, W., Noll, S., Smette, A., et al. 2015, A\&A, 576, A78

Kirkpatrick, J. D. 2005, ARA\&A, 43, 195

Kirkpatrick, J. D., Gelino, C. R., Cushing, M. C., et al. 2012, ApJ, 753, 156

Kopytova, T. G., Crossfield, I. J. M., Deacon, N. R., et al. 2014, ApJ, 797, 3

Kouwenhoven, M. B. N., Brown, A. G. A., Zinnecker, H., Kaper, L., \& Portegies Zwart, S. F. 2005, A\&A, 430, 137

Kraus, A. L., \& Hillenbrand, L. A. 2008, ApJ, 686, L111

Kraus, A. L., \& Hillenbrand, L. A. 2009, ApJ, 703, 1511

Kraus, A. L., \& Hillenbrand, L. A. 2012, ApJ, 757, 141

Kraus, A. L., Ireland, M. J., Martinache, F., \& Lloyd, J. P. 2008, ApJ, 679, 762

Kraus, A. L., Ireland, M. J., Cieza, L. A., et al. 2014, ApJ, 781, 20

Lachapelle, F.-R., Lafrenière, D., Gagné, J., et al. 2015, ApJ, 802, 61

Lafrenière, D., Jayawardhana, R., \& van Kerkwijk, M. H. 2008, ApJ, 689, L153

Lafrenière, D., Jayawardhana, R., \& van Kerkwijk, M. H. 2010, ApJ, 719, 497

Lafrenière, D., Jayawardhana, R., Janson, M., et al. 2011, ApJ, 730, 42

Lallement, R., Babusiaux, C., Vergely, J. L., et al. 2019, A\&A, 625, A135

Lindegren, L., Hernández, J., Bombrun, A., et al. 2018, A\&A, 616, A2

Lodieu, N., Hambly, N. C., Jameson, R. F., et al. 2007, MNRAS, 374, 372

Lodieu, N., Hambly, N. C., Jameson, R. F., \& Hodgkin, S. T. 2008, MNRAS, 383,1385

Lodieu, N., Dobbie, P. D., \& Hambly, N. C. 2011, A\&A, 527, A24

Lodieu, N., Zapatero Osorio, M. R., Béjar, V. J. S., \& Peña Ramírez, K. 2018, MNRAS, 473, 2020

López-Morales, M. 2007, ApJ, 660, 732

Luhman, K. L., \& Mamajek, E. E. 2012, ApJ, 758, 31

Luhman, K. L., Stauffer, J. R., Muench, A. A., et al. 2003, ApJ, 593, 1093

Luhman, K. L., Herrmann, K. A., Mamajek, E. E., Esplin, T. L., \& Pecaut, M. J. 2018, AJ, 156, 76

MacGregor, M. A., Wilner, D. J., Czekala, I., et al. 2017, ApJ, 835, 17

Madhusudhan, N., Burrows, A., \& Currie, T. 2011, ApJ, 737, 34

Manara, C. F., Testi, L., Rigliaco, E., et al. 2013, A\&A, 551, A107

Manara, C. F., Testi, L., Natta, A., \& Alcalá, J. M. 2015, A\&A, 579, A66

Manjavacas, E., Bonnefoy, M., Schlieder, J. E., et al. 2014, A\&A, 564, A55
Marks, M., Martín, E. L., Béjar, V. J. S., et al. 2017, A\&A, 605, A11

Mayor, M., \& Queloz, D. 1995, Nature, 378, 355

McDonald, I., Zijlstra, A. A., \& Watson, R. A. 2017, MNRAS, 471, 770

Modigliani, A., Goldoni, P., Royer, F., et al. 2010, in Observatory Operations: Strategies, Processes, and Systems III (Bellingham, USA: SPIE), 7737, 773728

Morales, J. C., Ribas, I., \& Jordi, C. 2008, A\&A, 478, 507

Mordasini, C., Alibert, Y., \& Benz, W. 2009, A\&A, 501, 1139

Mordasini, C., Alibert, Y., Benz, W., Klahr, H., \& Henning, T. 2012, A\&A, 541, A97

Mordasini, C., Marleau, G.-D., \& Mollière, P. 2017, A\&A, 608, A72

Morley, C. V., Fortney, J. J., Marley, M. S., et al. 2012, ApJ, 756, 172

Nakajima, T., Oppenheimer, B. R., Kulkarni, S. R., et al. 1995, Nature, 378 , 463

Natta, A., Testi, L., Muzerolle, J., et al. 2004, A\&A, 424, 603

Neckel, T., \& Klare, G. 1980, A\&AS, 42, 251

Neuhäuser, R., Guenther, E. W., Petr, M. G., et al. 2000, A\&A, 360, L39

Padoan, P., \& Nordlund, A. 2004, ApJ, 617, 559

Pavlenko, Y. V., Jones, H. R. A., Martín, E. L., et al. 2007, MNRAS, 380, 1285

Pecaut, M. J., \& Mamajek, E. E. 2013, ApJS, 208, 9

Pecaut, M. J., Mamajek, E. E., \& Bubar, E. J. 2012, ApJ, 746, 154

Preibisch, T., Guenther, E., \& Zinnecker, H. 2001, AJ, 121, 1040

Raghavan, D., McAlister, H. A., Henry, T. J., et al. 2010, ApJS, 190, 1

Rajpurohit, A. S., Allard, F., Rajpurohit, S., et al. 2018, A\&A, 620, A180

Rebolo, R., Zapatero Osorio, M. R., \& Martín, E. L. 1995, Nature, 377, 129

Reid, I. N., Hawley, S. L., \& Gizis, J. E. 1995, AJ, 110, 1838

Reid, I. N., Cruz, K. L., Kirkpatrick, J. D., et al. 2008, AJ, 136, 1290

Riaz, B., \& Gizis, J. E. 2008, ApJ, 681, 1584

Rigliaco, E., Natta, A., Testi, L., et al. 2012, A\&A, 548, A56

Rodriguez, D. R., van der Plas, G., Kastner, J. H., et al. 2015, A\&A, 582, L5

Sahlmann, J., Ségransan, D., Queloz, D., et al. 2011, A\&A, 525, A95

Samland, M., Mollière, P., Bonnefoy, M., et al. 2017, A\&A, 603, A57

Santamaría-Miranda, A., Cáceres, C., Schreiber, M. R., et al. 2018, MNRAS, 475, 2994

Saumon, D., \& Marley, M. S. 2008, ApJ, 689, 1327

Schmidt, T. O. B., Neuhäuser, R., Seifahrt, A., et al. 2008, A\&A, 491, 311

Seifahrt, A., Neuhäuser, R., \& Hauschildt, P. H. 2007, A\&A, 463, 309

Skilling, J. 2006, Bayesian Anal., 1, 833

Slesnick, C. L., Hillenbrand, L. A., \& Carpenter, J. M. 2004, ApJ, 610, 1045

Smette, A., Sana, H., Noll, S., et al. 2015, A\&A, 576, A77

Stamatellos, D., \& Whitworth, A. P. 2009, MNRAS, 392, 413

Stassun, K. G., Kratter, K. M., Scholz, A., \& Dupuy, T. J. 2012, ApJ, 756, 47

Thies, I., Pflamm-Altenburg, J., Kroupa, P., \& Marks, M. 2015, ApJ, 800, 72

Tokovinin, A. 2014, AJ, 147, 87

Tremblin, P., Chabrier, G., Baraffe, I., et al. 2017, ApJ, 850, 46

Vernet, J., Dekker, H., D’Odorico, S., et al. 2011, A\&A, 536, A105

Wagner, K., Follete, K. B., Close, L. M., et al. 2018, ApJ, 863, L8

Walter, F. M., Vrba, F. J., Mathieu, R. D., Brown, A., \& Myers, P. C. 1994, AJ, 107,692

Wang, J., David, T. J., Hillenbrand, L. A., et al. 2018, ApJ, 865, 141

White, R. J., \& Basri, G. 2003, ApJ, 582, 1109

Wolff, S. G., Ménard, F., Caceres, C., et al. 2017, AJ, 154, 26

Wu, Y.-L., Close, L. M., Eisner, J. A., \& Sheehan, P. D. 2017a, AJ, 154, 234

Wu, Y.-L., Sheehan, P. D., Males, J. R., et al. 2017b, ApJ, 836, 223

Zhou, Y., Herczeg, G. J., Kraus, A. L., Metchev, S., \& Cruz, K. L. 2014, ApJ, 783, L17 


\section{Appendix A: Temperature and reddening of USco 1610-1913}

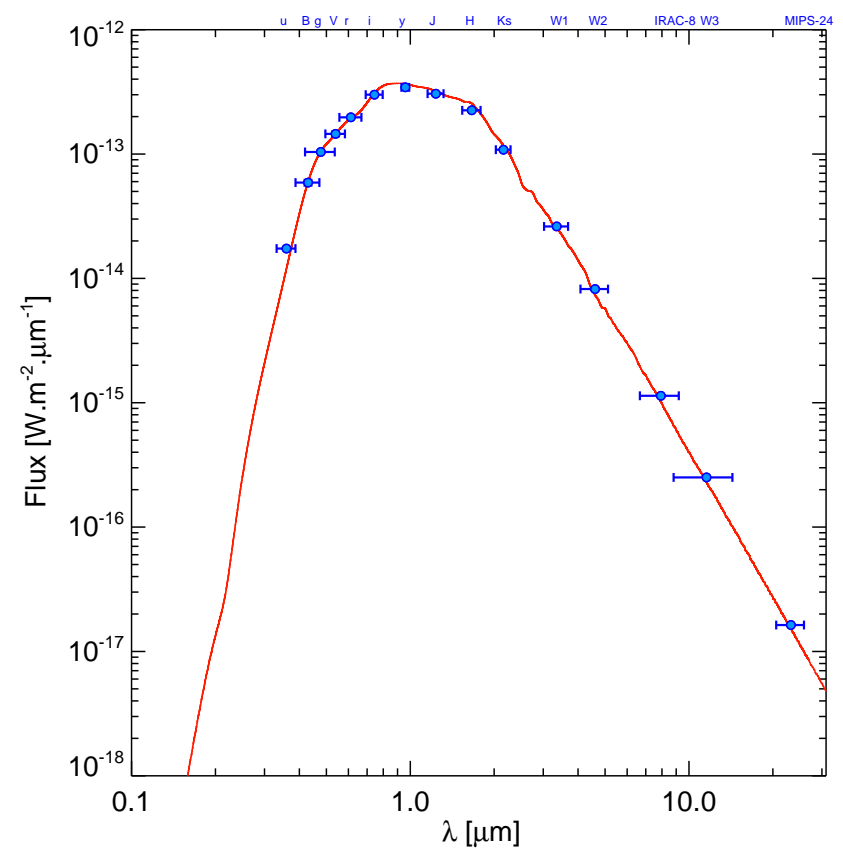

Fig. A.1. Spectral energy distribution of USco 1610-1913 dereddened by $A_{v}=0.09$ mag compared to the best-fitting BT-NEXTGEN model (red line) corresponding to $T_{\text {eff }}=3800 \mathrm{~K}, \log g=4.0 \mathrm{dex}$, and $M / H=0$ dex.

We built the SED of USco 1610-1913 from SDSS ( $u, g, r, i$ bands Alam et al. 2015), APASS ( $B$ and $V$ bands Henden et al. 2016), Pan-Starrs ( $y$ band Chambers et al. 2016), 2MASS (J, H, Ks, Cutri et al. 2003) and WISE (W1, W2, W3 bands Cutri et al. 2013) photometry collected through the VOSA (Bayo et al. 2008) web interface ${ }^{6}$ supplemented by Spitzer $(8,24 \mu \mathrm{m})$ photometry taken from Carpenter et al. (2006).

We compared deredenned SED of the object to synthetic spectra from the BT-NEXTGEN library (Allard et al. 2012) with $T_{\text {eff }}$ in the range $3500-4000 \mathrm{~K}$ and $M / H=0$. The surface-gravity was varied from 3.0 to 5.0 dex and does not influence the fit. We considered $A_{\mathrm{v}}$ in the range $0-5 \mathrm{mag}$ in steps of $0.01 \mathrm{mag}$ and a reddening law (Draine 2003a,c,b) with a reddening parameter $R_{\mathrm{v}}=A_{\mathrm{v}} / E(B-V)=3.1$.

We find a best fit for $T_{\text {eff }}=3800 \pm 100 \mathrm{~K}$ and $A_{\mathrm{v}}=0.09 \pm$ $0.01 \mathrm{mag}$ ) (Fig. A). The temperature is in broad agreement (Pecaut \& Mamajek 2013) with that expected for the quoted K7 spectral type (Preibisch et al. 2001) and rather suggests a M0 type for the object. We confirm the lack of an excess up to $24 \mu \mathrm{m}$. The fit clearly excludes solutions at a higher $T_{\text {eff }}$ and a reddening of $A_{\mathrm{v}}=1.1 \mathrm{mag}$ as reported by Carpenter et al. (2009).

\section{Appendix B: Systematic differences in spectral type determination}

To determine the spectral type of our sources we focused on the visible using a standard $\chi^{2}$ comparison between our spectra and the Ultracool RIZzo Spectral Library (see Sect. 4.1). We also used relations between the spectral type and the $\mathrm{H}_{2} \mathrm{O}$ absorption bands from Allers et al. (2007) $\left(\mathrm{H}_{2} \mathrm{O}(\mathrm{H})\right)$ and Slesnick et al. (2004) $\left(\mathrm{H}_{2} \mathrm{O}(\mathrm{J}) ; \mathrm{H}_{2} \mathrm{O}\right.$ K). Figure $\mathrm{B}$ shows the systematic differences according to each method used to determine the spectral type.

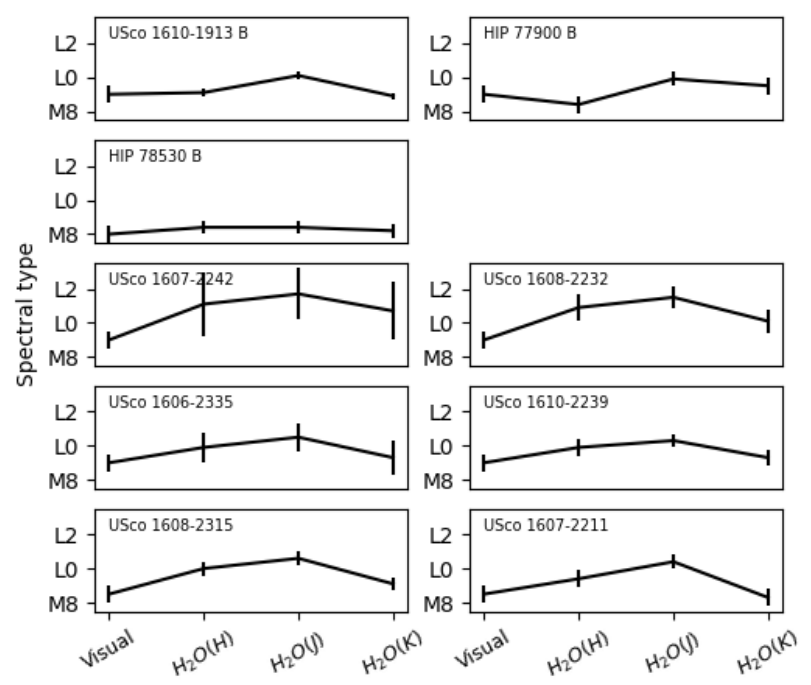

Fig. B.1. Determination of the spectral type in using $\chi^{2}$ comparison in optic and $\mathrm{H}_{2} \mathrm{O}$ absorption bands.

\footnotetext{
6 http://svo2.cab.inta-csic.es/theory/vosa/index.php
} 
Appendix C: Additional tables

Table C.1. Results from fits with ForMoSA.

\begin{tabular}{|c|c|c|c|c|c|c|c|c|c|}
\hline TWA 26 & $J$ & $H$ & $K$ & $J H K$ & $J H K$ and $A_{\mathrm{v}}$ & $J H K$-cont & OPT-cont & OPT-croscor & Adopted \\
\hline$T_{\text {eff }}(K)$ & $2412 \pm 1$ & $2640 \pm 1$ & $2590 \pm 1$ & $2114 \pm 1$ & $2587 \pm 1$ & $2534 \pm 2$ & $2522 \pm 3$ & - & $2547_{-136}^{+94}{ }^{(a)}$ \\
\hline $\log (g)(\operatorname{dex})$ & $4.10 \pm 0.01$ & 3.5 & 3.5 & 3.5 & 3.5 & $3.95 \pm 0.01$ & $4.32 \pm 0.01$ & - & $\leq 4.11^{(b)}$ \\
\hline$R\left(R_{\mathrm{Jup}}\right)$ & $2.61 \pm 0.01$ & $2.46 \pm 0.01$ & $2.83 \pm 0.01$ & $3.72 \pm 0.01$ & $3.10 \pm 0.01$ & NA & NA & - & $1.97_{-0.52}^{+0.87(a)}$ \\
\hline $\mathrm{RV}\left(\mathrm{km} \mathrm{s}^{-1}\right)$ & $-20.0 \pm 0.2$ & $10.2 \pm 0.2$ & $4.1 \pm 0.1$ & $15.1 \pm 0.1$ & $5.4 \pm 0.1$ & $1.4 \pm 0.1$ & $18.2 \pm 0.2$ & - & $18.2 \pm 0.2$ \\
\hline $\log \left(L / L_{\odot}\right)$ & $-2.68 \pm 0.01$ & $-2.57 \pm 0.01$ & $-2.48 \pm 0.01$ & $-2.60 \pm 0.01$ & $-2.41 \pm 0.01$ & NA & NA & - & $-2.83_{-0.36}^{+0.38(c)}$ \\
\hline$A_{\mathrm{v}}(\mathrm{mag})$ & \multicolumn{4}{|c|}{0.0} & $2.58 \pm 0.01$ & \multicolumn{2}{|c|}{0.0} & - & $0.0^{(d)}$ \\
\hline$v \sin (i)\left(\mathrm{km} \mathrm{s}^{-1}\right)$ & \multicolumn{5}{|c|}{-} & & $\leq 44$ & - & $\leq 44$ \\
\hline TWA 29 & $J$ & $H$ & $K$ & $J H K$ & $J H K$ and $A_{\mathrm{v}}$ & $J H K$-cont & OPT-cont & OPT-croscor & Adopted \\
\hline$T_{\text {eff }}(\mathrm{K})$ & $2425 \pm 2$ & $2577 \pm 3$ & $2564 \pm 5$ & $2133 \pm 1$ & $2554 \pm 2$ & $2519 \pm 7$ & $2495 \pm 7$ & - & $2522_{-99}^{+58}$ \\
\hline $\log (g)(\operatorname{dex})$ & $4.11 \pm 0.02$ & 3.5 & $4.31 \pm 0.02$ & 3.5 & 3.5 & $3.99 \pm 0.02$ & $4.29 \pm 0.03$ & - & $\leq 4.33$ \\
\hline$R\left(R_{\mathrm{Jup}}\right)$ & $2.08 \pm 0.01$ & $2.05 \pm 0.01$ & $2.28 \pm 0.01$ & $2.92 \pm 0.01$ & $2.48 \pm 0.01$ & NA & NA & - & $2.14_{-0.10}^{+0.15}$ \\
\hline $\mathrm{RV}\left(\mathrm{km} \mathrm{s}^{-1}\right)$ & $-21.0 \pm 1.0$ & $8.1 \pm 0.7$ & $-7.0 \pm 0.5$ & $-18.1 \pm 0.3$ & $-9.9 \pm 0.4$ & $-6.7 \pm 0.5$ & $3.2 \pm 1.0$ & - & $3.2 \pm 1.0$ \\
\hline $\log \left(L / L_{\odot}\right)$ & $-2.87 \pm 0.01$ & $-2.77 \pm 0.01$ & $-2.69 \pm 0.01$ & $-2.79 \pm 0.01$ & $-2.62 \pm 0.01$ & NA & NA & - & $-2.77_{-0.11}^{+0.10}$ \\
\hline$A_{\mathrm{v}}(\mathrm{mag})$ & \multicolumn{4}{|c|}{0.0} & $2.32 \pm 0.01$ & \multicolumn{2}{|c|}{0.0} & - & 0.0 \\
\hline$v \sin (i)\left(\mathrm{km} \mathrm{s}^{-1}\right)$ & \multicolumn{5}{|c|}{-} & & $66 \pm 2$ & - & $66 \pm 2$ \\
\hline USco 1610-1913 B & $J$ & $H$ & $K$ & $J H K$ & $J H K$ and $A_{\mathrm{v}}$ & $J H K$-cont & OPT-cont & OPT-croscor & Adopted \\
\hline$T_{\text {eff }}(\mathrm{K})$ & $2440 \pm 2$ & $2607 \pm 3$ & $2580 \pm 3$ & 2100 & $2652 \pm 2$ & $2654 \pm 8$ & $2565 \pm 4$ & - & $2542_{-104}^{+68}$ \\
\hline $\log (g)(\operatorname{dex})$ & $4.16 \pm 0.01$ & 3.5 & $3.94 \pm 0.02$ & 3.5 & $3.78 \pm 0.02$ & $3.94 \pm 0.02$ & $4.23 \pm 0.02$ & - & $\leq 4.17$ \\
\hline$R\left(R_{\mathrm{Jup}}\right)$ & $3.76 \pm 0.01$ & $3.76 \pm 0.01$ & $4.10 \pm 0.01$ & $5.12 \pm 0.01$ & $4.31 \pm 0.01$ & NA & NA & - & $3.87_{-0.12}^{+0.24}$ \\
\hline $\mathrm{RV}\left(\mathrm{km} \mathrm{s}^{-1}\right)$ & $18.9 \pm 0.9$ & $5.3 \pm 0.6$ & $-2.4 \pm 0.4$ & $13.2 \pm 0.2$ & $-2.2 \pm 0.3$ & $-0.1 \pm 0.2$ & $11.5 \pm 0.4$ & 0.9 & $11.5 \pm 0.4$ \\
\hline $\log \left(L / L_{\odot}\right)$ & $-2.34 \pm 0.01$ & $-2.23 \pm 0.01$ & $-2.17 \pm 0.01$ & $-2.26 \pm 0.01$ & $-2.08 \pm 0.01$ & NA & NA & - & $-2.25_{-0.10}^{+0.10}$ \\
\hline$A_{\mathrm{v}}(\mathrm{mag})$ & \multicolumn{4}{|c|}{0.13} & $2.24 \pm 0.01$ & \multicolumn{2}{|c|}{0.13} & - & 0.13 \\
\hline$v \sin (i)\left(\mathrm{km} \mathrm{s}^{-1}\right)$ & \multicolumn{5}{|c|}{-} & & $\leq 44$ & - & $\leq 44$ \\
\hline HIP $78530 B$ & $J$ & $H$ & $K$ & $J H K$ & $J H K$ and $A_{\mathrm{v}}$ & $J H K$-cont & OPT-cont & OPT-croscor & Adopted \\
\hline$T_{\text {eff }}(\mathrm{K})$ & $2566 \pm 6$ & $2791 \pm 6$ & $2681 \pm 8$ & $2590 \pm 1$ & $2772 \pm 3$ & $2713 \pm 13$ & $2619 \pm 14$ & - & $2679_{-119}^{+118}$ \\
\hline $\log (g)(\operatorname{dex})$ & $4.30 \pm 0.04$ & $4.00 \pm 0.03$ & $3.64 \pm 0.09$ & $4.05 \pm 0.01$ & $4.05 \pm 0.01$ & $4.27 \pm 0.04$ & $4.76 \pm 0.04$ & - & $\leq 4.34$ \\
\hline$R\left(R_{\mathrm{Jup}}\right)$ & $1.81 \pm 0.01$ & $1.70 \pm 0.01$ & $1.98 \pm 0.01$ & $1.93 \pm 0.01$ & $2.00 \pm 0.01$ & NA & NA & - & $1.83_{-0.14}^{+0.16}$ \\
\hline $\mathrm{RV}\left(\mathrm{km} \mathrm{s}^{-1}\right)$ & $-14.0 \pm 2.2$ & $-2.0 \pm 1.3$ & $-10.7 \pm 1.2$ & $-7.8 \pm 0.7$ & $-3.0 \pm 0.9$ & $-4.2 \pm 0.9$ & $7.5 \pm 1.0$ & 8.5 & $7.5 \pm 1.0$ \\
\hline $\log \left(L / L_{\odot}\right)$ & $-2.89 \pm 0.01$ & $-2.80 \pm 0.01$ & $-2.74 \pm 0.01$ & $-2.82 \pm 0.01$ & $-2.67 \pm 0.01$ & NA & NA & - & $-2.87_{-0.15}^{+0.15}$ \\
\hline$A_{\mathrm{v}}(\mathrm{mag})$ & \multirow{2}{*}{\multicolumn{4}{|c|}{0.075}} & $1.97 \pm 0.01$ & \multicolumn{2}{|c|}{0.075} & - & 0.075 \\
\hline$v \sin (i)\left(\mathrm{km} \mathrm{s}^{-1}\right)$ & & & & & - & & $\leq 50$ & - & $\leq 50$ \\
\hline HIP $77900 \mathrm{~B}$ & $J$ & $H$ & $K$ & $J H K$ & $J H K$ and $A_{\mathrm{v}}$ & $J H K$-cont & OPT-cont & OPT-croscor & Adopted \\
\hline$T_{\mathrm{eff}}(\mathrm{K})$ & $2511 \pm 6$ & $2713 \pm 6$ & $2583 \pm 13$ & $2570 \pm 1$ & $2713 \pm 3$ & $2688 \pm 17$ & $2604 \pm 13$ & - & $2602_{-97}^{+117}$ \\
\hline $\log (g)(\operatorname{dex})$ & $4.23 \pm 0.04$ & $3.58 \pm 0.05$ & $4.27 \pm 0.09$ & $4.12 \pm 0.01$ & $4.03 \pm 0.02$ & $4.13 \pm 0.04$ & $4.59 \pm 0.05$ & - & $\leq 4.36$ \\
\hline$R\left(R_{\mathrm{Jup}}\right)$ & $1.73 \pm 0.01$ & $1.65 \pm 0.01$ & $1.90 \pm 0.01$ & $1.79 \pm 0.01$ & $1.88 \pm 0.01$ & NA & NA & - & $1.76_{-0.12}^{+0.15}$ \\
\hline $\mathrm{RV}\left(\mathrm{km} \mathrm{s}^{-1}\right)$ & $-22.7 \pm 2.3$ & $-22.7 \pm 1.3$ & $-29.5 \pm 1.6$ & $-23.9 \pm 0.8$ & $-22.8 \pm 0.9$ & $-22.9 \pm 0.8$ & $19.3 \pm 1.2$ & 20.1 & $19.3 \pm 1.2$ \\
\hline $\log \left(L / L_{\odot}\right)$ & $-2.97 \pm 0.01$ & $-2.87 \pm 0.01$ & $-2.84 \pm 0.01$ & $-2.90 \pm 0.01$ & $-2.76 \pm 0.01$ & NA & NA & - & $-2.89_{-0.13}^{+0.15}$ \\
\hline$A_{\mathrm{v}}(\mathrm{mag})$ & & & 07 & & $1.76 \pm 0.01$ & & 07 & - & 0.07 \\
\hline$v \sin (i)\left(\mathrm{km} \mathrm{s}^{-1}\right)$ & & & - & & & & $\leq 44$ & - & $\leq 44$ \\
\hline
\end{tabular}

Notes. ${ }^{(a)}$ Mean and standard deviation between $J, H$ and $K$ bands. ${ }^{(b)}$ High value between $J, H, K$ and $J H K$-cont bands. ${ }^{(c)}$ Stefan-Boltzmann law in using $T_{\text {eff }}$ and radius ranges. ${ }^{(d)}$ From Lallement et al. (2019)'s maps (derived from Galli et al. 2018). 
S. Petrus et al.: X-shooter view of wide orbit companion in Upper-Sco

Table C.2. Results from fits with ForMoSA.

\begin{tabular}{|c|c|c|c|c|c|c|c|c|c|}
\hline USco 1606-2335 & $J$ & $H$ & $K$ & $J H K$ & $J H K$ and $A_{\mathrm{v}}$ & $J H K$-cont & OPT-cont & OPT-croscor & Adopted \\
\hline$T_{\text {eff }}(\mathrm{K})$ & $2390 \pm 12$ & $2620 \pm 13$ & $2547 \pm 18$ & $2350 \pm 5$ & $2650 \pm 7$ & $2574 \pm 38$ & $2414 \pm 45$ & - & $2519_{-141}^{+114}$ \\
\hline $\log (g)(\operatorname{dex})$ & $4.13 \pm 0.08$ & 3.5 & 3.5 & 3.5 & $3.89 \pm 0.06$ & $4.28 \pm 0.08$ & 5.0 & - & $\leq 4.36$ \\
\hline$R\left(R_{\mathrm{Jup}}\right)$ & $1.41 \pm 0.01$ & $1.37 \pm 0.01$ & $1.59 \pm 0.02$ & $1.65 \pm 0.01$ & $1.66 \pm 0.01$ & NA & NA & - & $1.46_{-0.10}^{+0.15}$ \\
\hline $\mathrm{RV}\left(\mathrm{km} \mathrm{s}^{-1}\right)$ & $-11.7 \pm 4.9$ & $-0.9 \pm 1.6$ & $-10.0 \pm 2.8$ & $-10.1 \pm 1.2$ & $-3.4 \pm 1.5$ & $-4.3 \pm 1.6$ & $13.6 \pm 3.6$ & 13.1 & $10.1 \pm 4.1$ \\
\hline $\log \left(L / L_{\odot}\right)$ & $-3.23 \pm 0.01$ & $-3.09 \pm 0.01$ & $-3.01 \pm 0.01$ & $-3.12 \pm 0.01$ & $-2.91 \pm 0.01$ & NA & NA & - & $-3.11_{-0.16}^{+0.16}$ \\
\hline$A_{\mathrm{v}}(\mathrm{mag})$ & \multicolumn{4}{|c|}{0.0} & $3.06 \pm 0.03$ & \multicolumn{2}{|c|}{0.0} & - & $0.0^{(d)}$ \\
\hline$v \sin (i)\left(\mathrm{km} \mathrm{s}^{-1}\right)$ & \multicolumn{5}{|c|}{-} & & $\leq 45$ & - & $\leq 45$ \\
\hline USco 1607-2211 & $J$ & $H$ & $K$ & $J H K$ & $J H K$ and $A_{\mathrm{v}}$ & $J H K$-cont & OPT-cont & OPT-croscor & Adopted \\
\hline$T_{\text {eff }}(\mathrm{K})$ & $2445 \pm 5$ & $2616 \pm 6$ & $2609 \pm 5$ & $2384 \pm 1$ & $2621 \pm 2$ & $2674 \pm 13$ & $2532 \pm 15$ & - & $2557_{-117}^{+65}$ \\
\hline $\log (g)(\operatorname{dex})$ & $3.92 \pm 0.07$ & 3.5 & $3.79 \pm 0.06$ & 3.5 & $4.20 \pm 0.01$ & $4.02 \pm 0.03$ & $4.25 \pm 0.06$ & - & $\leq 4.05$ \\
\hline$R\left(R_{\mathrm{Jup}}\right)$ & $1.92 \pm 0.01$ & $1.87 \pm 0.01$ & $1.96 \pm 0.01$ & $2.18 \pm 0.01$ & $2.07 \pm 0.01$ & NA & NA & - & $1.92_{-0.06}^{+0.05}$ \\
\hline $\mathrm{RV}\left(\mathrm{km} \mathrm{s}^{-1}\right)$ & $-31.0 \pm 1.8$ & $-28.2 \pm 1.1$ & $-0.5 \pm 0.6$ & $-14.2 \pm 0.4$ & $-8.3 \pm 0.5$ & $-9.1 \pm 0.5$ & $-10.1 \pm 1.3$ & -10.2 & $-11.1 \pm 1.4$ \\
\hline $\log \left(L / L_{\odot}\right)$ & $-2.92 \pm 0.01$ & $-2.83 \pm 0.01$ & $-2.79 \pm 0.01$ & $-2.85 \pm 0.01$ & $-2.74 \pm 0.01$ & NA & NA & - & $-2.84_{-0.11}^{+0.07}$ \\
\hline$A_{\mathrm{v}}(\mathrm{mag})$ & \multirow{2}{*}{\multicolumn{4}{|c|}{0.0}} & $1.64 \pm 0.01$ & \multicolumn{2}{|c|}{0.0} & - & 0.0 \\
\hline$v \sin (i)\left(\mathrm{km} \mathrm{s}^{-1}\right)$ & & & & & & & $\leq 45$ & - & $\leq 45$ \\
\hline USco 1607-2242 & $J$ & $H$ & $K$ & $J H K$ & $J H K$ and $A_{\mathrm{v}}$ & $J H K$-cont & OPT-cont & OPT-croscor & Adopted \\
\hline$T_{\text {eff }}(\mathrm{K})$ & $2265 \pm 14$ & $2512 \pm 13$ & $2432 \pm 23$ & 2100 & $2579 \pm 8$ & $2432 \pm 71$ & $2380 \pm 102$ & - & $2403_{-152}^{+122}$ \\
\hline $\log (g)(\operatorname{dex})$ & $3.84 \pm 0.07$ & 3.5 & 3.5 & 3.5 & 3.5 & $3.96 \pm 0.14$ & $4.01 \pm 0.27$ & - & $\leq 4.10$ \\
\hline$R\left(R_{\text {Jup }}\right)$ & $1.09 \pm 0.02$ & $1.06 \pm 0.01$ & $1.24 \pm 0.02$ & $1.47 \pm 0.01$ & $1.31 \pm 0.01$ & NA & NA & - & $1.13_{-0.08}^{+0.13}$ \\
\hline $\mathrm{RV}\left(\mathrm{km} \mathrm{s}^{-1}\right)$ & $-19.0 \pm 6.1$ & $-0.1 \pm 1.2$ & $-5.3 \pm 3.3$ & $-9.9 \pm 1.2$ & $-1.1 \pm 1.4$ & $-1.1 \pm 1.5$ & $6.7 \pm 11.6$ & 9.6 & $5.7 \pm 10.8$ \\
\hline $\log \left(L / L_{\odot}\right)$ & $-3.55 \pm 0.01$ & $-3.39 \pm 0.01$ & $-3.31 \pm 0.01$ & $-3.42 \pm 0.01$ & $-3.16 \pm 0.01$ & NA & NA & - & $-3.41_{-0.18}^{+0.18}$ \\
\hline$A_{\mathrm{v}}(\mathrm{mag})$ & \multicolumn{4}{|c|}{0.0} & $3.82 \pm 0.04$ & \multicolumn{2}{|c|}{0.0} & - & 0.0 \\
\hline$v \sin (i)\left(\mathrm{km} \mathrm{s}^{-1}\right)$ & \multicolumn{5}{|c|}{-} & & $\leq 45$ & - & $\leq 45$ \\
\hline USco 1608-2232 & $J$ & $H$ & $K$ & $J H K$ & $J H K$ and $A_{\mathrm{v}}$ & $J H K$-cont & T-cont & OPT-croscor & Adopted \\
\hline$T_{\text {eff }}(\mathrm{K})$ & $2317 \pm 7$ & $2482 \pm 8$ & $2427 \pm 12$ & 2100 & $2555 \pm 5$ & $2510 \pm 26$ & $2363 \pm 50$ & - & $2409_{-99}^{+81}$ \\
\hline $\log (g)(\operatorname{dex})$ & $3.85 \pm 0.05$ & 3.5 & 3.5 & 3.5 & 3.5 & $4.01 \pm 0.08$ & 5.0 & - & $\leq 4.09$ \\
\hline$R\left(R_{\mathrm{Jup}}\right)$ & $1.52 \pm 0.01$ & $1.56 \pm 0.01$ & $1.81 \pm 0.01$ & $2.13 \pm 0.01$ & $1.91 \pm 0.01$ & NA & NA & - & $1.63_{-0.12}^{+0.19}$ \\
\hline $\mathrm{RV}\left(\mathrm{km} \mathrm{s}^{-1}\right)$ & $-14.1 \pm 3.6$ & $-0.5 \pm 1.2$ & $-3.5 \pm 1.9$ & $-10.5 \pm 0.8$ & $-1.4 \pm 1.1$ & $-1.5 \pm 1.2$ & $11.1 \pm 4.0$ & 9.7 & $11.0 \pm 4.5$ \\
\hline $\log \left(L / L_{\odot}\right)$ & $-3.22 \pm 0.01$ & $-3.08 \pm 0.01$ & $-2.99 \pm 0.01$ & $-3.10 \pm 0.01$ & $-2.85 \pm 0.01$ & NA & NA & - & $-3.09_{-0.14}^{+0.15}$ \\
\hline$A_{\mathrm{v}}(\mathrm{mag})$ & \multirow{2}{*}{\multicolumn{4}{|c|}{0.0}} & $3.65 \pm 0.02$ & \multicolumn{2}{|c|}{0.0} & - & 0.0 \\
\hline$v \sin (i)\left(\mathrm{km} \mathrm{s}^{-1}\right)$ & & & & & & & $\leq 47$ & - & $\leq 47$ \\
\hline USco 1608-2315 & $J$ & $H$ & $K$ & $I H K$ & $J H K$ and $A_{\mathrm{v}}$ & $J H K$-cont & OPT-cont & OPT-cro & Adopted \\
\hline$T_{\text {eff }}(\mathrm{K})$ & $2344 \pm 4$ & $2555 \pm 5$ & $2561 \pm 7$ & 2100 & $2659 \pm 3$ & $2664 \pm 15$ & $2494 \pm 27$ & - & $2487_{-147}^{+81}$ \\
\hline $\log (g)(\operatorname{dex})$ & $3.80 \pm 0.03$ & 3.5 & 3.5 & 3.5 & $3.66 \pm 0.02$ & $4.13 \pm 0.03$ & $4.27 \pm 0.10$ & - & $\leq 4.16$ \\
\hline$R\left(R_{\mathrm{Jup}}\right)$ & $1.96 \pm 0.01$ & $1.92 \pm 0.01$ & $2.11 \pm 0.01$ & $2.78 \pm 0.01$ & $2.26 \pm 0.01$ & NA & NA & - & $2.00_{-0.09}^{+0.12}$ \\
\hline $\mathrm{RV}\left(\mathrm{km} \mathrm{s}^{-1}\right)$ & $-15.4 \pm 2.1$ & $-0.1 \pm 0.5$ & $-1.8 \pm 1.0$ & $-13.8 \pm 0.4$ & $-0.2 \pm 0.3$ & $-0.1 \pm 0.2$ & $10.6 \pm 2.3$ & 5.3 & $10.4 \pm 2.5$ \\
\hline $\log \left(L / L_{\odot}\right)$ & $-2.98 \pm 0.01$ & $-2.84 \pm 0.01$ & $-2.76 \pm 0.01$ & $-2.86 \pm 0.01$ & $-2.63 \pm 0.01$ & NA & NA & - & $-2.86_{-0.15}^{+0.11}$ \\
\hline$A_{\mathrm{v}}(\mathrm{mag})$ & \multicolumn{4}{|c|}{0.0} & $3.28 \pm 0.01$ & \multicolumn{2}{|c|}{0.0} & - & 0.0 \\
\hline$v \sin (i)\left(\mathrm{km} \mathrm{s}^{-1}\right)$ & \multicolumn{5}{|c|}{ - } & & $\leq 45$ & - & $\leq 45$ \\
\hline USco 1610-2239 & $J$ & $H$ & $K$ & $J H K$ & $J H K$ and $A_{\mathrm{v}}$ & $J H K$-cont & OPT-cont & OPT-croscor & Adopted \\
\hline$T_{\text {eff }}(\mathrm{K})$ & $2397 \pm 6$ & $2596 \pm 5$ & $2503 \pm 7$ & $2343 \pm 2$ & $2633 \pm 3$ & $2612 \pm 16$ & $2514 \pm 19$ & - & $2499_{-108}^{+102}$ \\
\hline $\log (g)(\operatorname{dex})$ & $3.87 \pm 0.05$ & 3.5 & 3.5 & 3.5 & 3.5 & $3.97 \pm 0.04$ & $4.31 \pm 0.08$ & - & $\leq 4.01$ \\
\hline$R\left(R_{\mathrm{Jup}}\right)$ & $1.87 \pm 0.01$ & $1.85 \pm 0.01$ & $2.15 \pm 0.01$ & $2.21 \pm 0.01$ & $2.21 \pm 0.01$ & NA & NA & - & $1.98_{-0.14}^{+0.24}$ \\
\hline $\mathrm{RV}\left(\mathrm{km} \mathrm{s}^{-1}\right)$ & $-13.0 \pm 2.1$ & $-0.1 \pm 0.4$ & $-3.4 \pm 1.1$ & $-7.6 \pm 0.5$ & $-0.9 \pm 0.6$ & $-0.5 \pm 0.6$ & $7.7 \pm 1.7$ & 7.9 & $11.3 \pm 1.8$ \\
\hline $\log \left(L / L_{\odot}\right)$ & $-2.98 \pm 0.01$ & $-2.85 \pm 0.01$ & $-2.78 \pm 0.01$ & $-2.87 \pm 0.01$ & $-2.67 \pm 0.01$ & NA & NA & - & $-2.86_{-0.14}^{+0.17}$ \\
\hline$A_{\mathrm{v}}(\mathrm{mag})$ & \multirow{2}{*}{\multicolumn{4}{|c|}{0.0}} & $2.91 \pm 0.01$ & \multicolumn{2}{|c|}{0.0} & - & 0.0 \\
\hline$v \sin (i)\left(\mathrm{km} \mathrm{s}^{-1}\right)$ & & & & & & & $\leq 45$ & - & $\leq 45$ \\
\hline
\end{tabular}


Table C.3. Apparent fluxes of the emission lines for the companions and free-floating brown dwarfs from our original sample.

\begin{tabular}{|c|c|c|c|c|c|c|c|}
\hline Object & Date & $\begin{array}{c}\text { Ca II-H } \\
\left(10^{-19} \mathrm{~W} \mathrm{~m}^{-2}\right)\end{array}$ & $\begin{array}{c}\text { Ca II-K } \\
\left(10^{-19} \mathrm{~W} \mathrm{~m}^{-2}\right)\end{array}$ & $\begin{array}{c}\mathrm{H}_{\gamma} \\
\left(10^{-19} \mathrm{~W} \mathrm{~m}^{-2}\right)\end{array}$ & $\begin{array}{c}\mathrm{H}_{\beta} \\
\left(10^{-19} \mathrm{~W} \mathrm{~m}^{-2}\right)\end{array}$ & $\begin{array}{c}\mathrm{H}_{\alpha} \\
\left(10^{-19} \mathrm{~W} \mathrm{~m}^{-2}\right)\end{array}$ & $\begin{array}{l}10 \% \mathrm{H}_{\alpha} \\
\left(\mathrm{km} \mathrm{s}^{-1}\right)\end{array}$ \\
\hline USco 1610-1913 B & 2014-04-02 & $0.27 \pm 0.11$ & $0.23 \pm 0.15$ & $0.23 \pm 0.18$ & $0.56 \pm 0.21$ & $4.86 \pm 0.26$ & 145 \\
\hline HIP $77900 \mathrm{~B}$ & 2014-04-12 05:45UT & $<0.31$ & $<0.22$ & $<0.45$ & $<0.46$ & $0.40 \pm 0.15$ & $\ldots$ \\
\hline HIP $77900 \mathrm{~B}$ & 2014-04-12 08:38UT & $<0.20$ & $<0.23$ & $<0.24$ & $<0.37$ & $0.58 \pm 0.15$ & $\ldots$ \\
\hline HIP 78530 B & 2014-06-09 04:08UT & $<0.95$ & $<0.99$ & $<1.28$ & $<2.61$ & $0.68 \pm 0.36$ & $\ldots$ \\
\hline HIP 78530 B & 2014-06-09 05:14UT & $<0.47$ & $<0.72$ & $<0.79$ & $<2.19$ & $0.61 \pm 0.29$ & $\ldots$ \\
\hline USco 1607-2242 & all epochs & $<0.06$ & $<0.10$ & $<0.07$ & $<0.09$ & $<0.10$ & \\
\hline USco 1608-2232 & all epochs & $<0.04$ & $<0.04$ & $<0.03$ & $<0.10$ & $<0.14$ & $\ldots$ \\
\hline USco 1606-2335 & $2014-06-25$ & $<0.15$ & $<0.13$ & $0.14 \pm 0.14$ & $0.23 \pm 0.17$ & $0.53 \pm 0.19$ & $\ldots$ \\
\hline USco 1606-2335 & 2014-06-30 & $<0.09$ & $<0.32$ & $0.22 \pm 0.19$ & $0.18 \pm 0.11$ & $0.72 \pm 0.17$ & 169 \\
\hline USco 1606-2335 & 2014-07-04 & $<0.07$ & $<0.09$ & $0.24 \pm 0.12$ & $0.39 \pm 0.11$ & $1.58 \pm 0.15$ & 136 \\
\hline USco 1606-2335 & 2014-07-29 & $<0.08$ & $<0.09$ & $0.18 \pm 0.10$ & $0.22 \pm 0.08$ & $0.85 \pm 0.15$ & 105: \\
\hline USco 1610-2239 & 2014-06-30 & $<0.10$ & $<0.10$ & $<0.15$ & $<0.13$ & $0.28 \pm 0.09$ & $\ldots$ \\
\hline USco 1610-2239 & 2014-07-01 03:26UT & $<0.16$ & $<0.93$ & $<0.19$ & $<0.19$ & $0.41 \pm 0.16$ & $\ldots$ \\
\hline USco 1610-2239 & 2014-07-01 04:04UT & $<0.07$ & $<0.11$ & $<0.13$ & $<0.12$ & $0.39 \pm 0.11$ & $\ldots$ \\
\hline USco 1608-2315 & 2014-06-15 & $<0.11$ & $<0.29$ & $1.07 \pm 0.23$ & $1.85 \pm 0.25$ & $11.99 \pm 0.38$ & 220 \\
\hline USco 1608-2315 & 2014-06-20 & $<0.11$ & $<0.25$ & $0.83 \pm 0.14$ & $1.07 \pm 0.14$ & $7.65 \pm 0.30$ & 209 \\
\hline USco 1607-2211 & 2014-04-22 & $<0.17$ & $<0.15$ & $<0.20$ & $<0.23$ & $0.99 \pm 0.18$ & 160 \\
\hline USco 1607-2211 & 2014-06-14 & $<0.11$ & $<0.11$ & $<0.17$ & $<0.23$ & $\ldots$ & $\ldots$ \\
\hline
\end{tabular}

Notes. We also report the $10 \%$ width of the $\mathrm{H}_{\alpha}$ line. The upper limits on the line flux correspond to the $1 \sigma$ noise level of the continuum.

Table C.4. Accretion luminosities computed from the emission lines.

\begin{tabular}{|c|c|c|c|c|c|c|c|c|}
\hline Object & Date & $\begin{array}{c}\text { Ca II-H } \\
\log \left(L_{\mathrm{acc}} / L_{\odot}\right)\end{array}$ & $\begin{array}{c}\text { Ca II-K } \\
\log \left(L_{\text {acc }} / L_{\odot}\right)\end{array}$ & $\begin{array}{c}\mathrm{H}_{\gamma} \\
\log \left(L_{\mathrm{acc}} / L_{\odot}\right)\end{array}$ & $\begin{array}{c}\mathrm{H}_{\beta} \\
\log \left(L_{\mathrm{acc}} / L_{\odot}\right)\end{array}$ & $\begin{array}{c}\mathrm{H}_{\alpha} \\
\log \left(L_{\mathrm{acc}} / L_{\odot}\right)\end{array}$ & $\begin{array}{c}\text { Balmer } \\
\log \left(L_{\mathrm{acc}} / L_{\odot}\right)\end{array}$ & $\log \left(L_{\text {acc,noise }} / L_{\odot}\right)$ \\
\hline $\begin{array}{l}\text { USco 1610-1913 B } \\
\text { HIP 77900 B } \\
\text { HIP 77900 B } \\
\text { HIP 78530 B } \\
\text { HIP 78530 B }\end{array}$ & $\begin{array}{c}\text { 2014-04-02 } \\
\text { 2014-04-12 05:45UT } \\
\text { 2014-04-12 08:38UT } \\
\text { 2014-06-09 04:08UT } \\
2014-06-0905: 14 \mathrm{UT}\end{array}$ & $\begin{array}{c}-5.57 \pm 0.21 \\
\leq-5.52 \\
\leq-5.73 \\
\leq-5.04 \\
\leq-5.36\end{array}$ & $\begin{array}{c}-5.56 \pm 0.37 \\
\leq-5.65 \\
\leq-5.63 \\
\leq-4.95 \\
\leq-5.09\end{array}$ & $\begin{aligned}-6.00 \pm 0.70 & \leq-5.67 \\
& \leq-5.98 \\
& \leq-5.22 \\
& \leq-5.45\end{aligned}$ & $\begin{array}{c}-5.89 \pm 0.14 \\
\leq-6.06 \\
\leq-6.05 \\
\leq-5.18 \\
\leq-5.26\end{array}$ & $\begin{array}{l}-5.61 \pm 0.05 \\
-6.79 \pm 0.05 \\
-6.61 \pm 0.02 \\
-6.62 \pm 0.18 \\
-6.67 \pm 0.13\end{array}$ & $\begin{array}{l}-5.70 \pm 0.30 \\
-6.79 \pm 0.05 \\
-6.61 \pm 0.02 \\
-6.62 \pm 0.18 \\
-6.67 \pm 0.13\end{array}$ & $\begin{array}{l}-5.65 \\
-6.06 \\
-6.07 \\
-6.07 \\
-6.07\end{array}$ \\
\hline USco 1607-2242 & all epochs & $\leq-6.26$ & $\leq-5.93$ & $\leq-6.56$ & $\leq-6.79$ & $\leq-7.51$ & & $\ldots$ \\
\hline USco 1608-2232 & all epochs & $\leq-6.45$ & $\leq-6.34$ & $\leq-6.97$ & $\leq-6.74$ & $\leq-7.34$ & & $\ldots$ \\
\hline USco $1606-2335$ & $2014-06-25$ & $\leq-5.84$ & $\leq-5.81$ & $-6.20 \pm 1.34$ & $-6.33 \pm 0.63$ & $-6.69 \pm 0.16$ & $-6.58 \pm 0.71$ & -6.60 \\
\hline USco 1606-2335 & 2014-06-30 & $\leq-6.07$ & $\leq-5.41$ & $-6.01 \pm 1.01$ & $-6.45 \pm 0.44$ & $-6.54 \pm 0.09$ & $-6.49 \pm 0.51$ & -6.60 \\
\hline USco 1606-2335 & 2014-07-04 & $\leq-6.19$ & $\leq-5.98$ & $-5.97 \pm 0.40$ & $-6.06 \pm 0.16$ & $-6.15 \pm 0.03$ & $-6.13 \pm 0.20$ & -6.60 \\
\hline USco 1606-2335 & 2014-07-29 & $\leq-6.13$ & $\leq-5.98$ & $-6.11 \pm 0.45$ & $-6.35 \pm 0.20$ & $-6.46 \pm 0.06$ & $-6.40 \pm 0.24$ & -6.60 \\
\hline USco 1610-2239 & 2014-06-30 & $\leq-6.13$ & $\leq-6.04$ & $\leq-6.31$ & $\leq-6.73$ & $-7.12 \pm 0.09$ & $-7.12 \pm 0.09$ & -6.33 \\
\hline USco $1610-2239$ & 2014-07-01 03:26UT & $\leq-5.92$ & $\leq-5.04$ & $\leq-6.20$ & $\leq-6.54$ & $-6.93 \pm 0.15$ & $-6.93 \pm 0.15$ & -6.33 \\
\hline USco 1610-2239 & 2014-07-01 04:04UT & $\leq-6.30$ & $\leq-5.99$ & $\leq-6.38$ & $\leq-6.77$ & $-6.96 \pm 0.07$ & $-6.96 \pm 0.07$ & -6.33 \\
\hline USco $1608-2315$ & 2014-06-15 & $\leq-5.98$ & $\leq-5.45$ & $-5.25 \pm 0.21$ & $-5.29 \pm 0.09$ & $-5.16 \pm 0.03$ & $-5.20 \pm 0.11$ & -6.30 \\
\hline USco $1608-2315$ & 2014-06-20 & $\leq-5.98$ & $\leq-5.52$ & $-5.37 \pm 0.18$ & $-5.56 \pm 0.08$ & $-6.34 \pm 0.04$ & $-5.99 \pm 0.10$ & -6.30 \\
\hline USco 1607-2211 & 2014-04-22 & $\leq-5.96$ & $\leq-5.92$ & $\leq-6.25$ & $\leq-6.52$ & $-6.57 \pm 0.07$ & $-6.57 \pm 0.07$ & -6.27 \\
\hline USco $1607-2211$ & 2014-06-14 & $\leq-6.16$ & $\leq-6.06$ & $\leq-6.32$ & $\leq-6.52$ & $\ldots$ & $\ldots$ & $\ldots$ \\
\hline
\end{tabular}

Notes. The accretion luminosity for each line is derived from Rigliaco et al. (2012). The weighted mean of the $L_{\text {acc }}$ values are reported in the "Balmer" column. The $\log \left(L_{\text {acc,noise }} / L_{\odot}\right)$ value of USco 1610-1913 B assumes the companion is a single object. In case of higher multiplicity, and assuming a $L_{\text {bol }}$ similar to HIP $77900 \mathrm{~B}$, we find $\log \left(L_{\text {acc,noise }} / L_{\odot}\right)=-6.11_{-0.22}^{+0.19}$. 
S. Petrus et al.: X-shooter view of wide orbit companion in Upper-Sco

Table C.5. Accretion rates from apparent fluxes of the emission lines for the companions and free-floating brown dwarfs from our original sample.

\begin{tabular}{cccccccc}
\hline \hline Object & Date & $\begin{array}{c}\mathrm{Ca} \mathrm{II-H} \\
\log \left(M_{\odot} \mathrm{yr}^{-1}\right)\end{array}$ & $\begin{array}{c}\mathrm{Ca} \mathrm{II-K} \\
\log \left(M_{\odot} \mathrm{yr}^{-1}\right)\end{array}$ & $\begin{array}{c}\mathrm{H}_{\gamma} \\
\log \left(M_{\odot} \mathrm{yr}^{-1}\right)\end{array}$ & $\begin{array}{c}\mathrm{H}_{\beta} \\
\log \left(M_{\odot} \mathrm{yr}^{-1}\right)\end{array}$ & $\begin{array}{c}\mathrm{H}_{\alpha} \\
\log \left(M_{\odot} \mathrm{yr}^{-1}\right)\end{array}$ & $\begin{array}{c}10 \% \mathrm{H}_{\alpha} \\
\log \left(M_{\odot} \mathrm{yr}^{-1}\right)\end{array}$ \\
\hline USco 1610-1913 B & $2014-04-02$ & $-12.10 \pm 0.53$ & $-12.09 \pm 0.69$ & $-12.53 \pm 1.02$ & $-12.42 \pm 0.46$ & $-12.14 \pm 0.37$ & $-11.48 \pm 0.40$ \\
HIP 77900 B & $2014-04-12$ 05:45UT & $<-11.69$ & $<-11.82$ & $<-11.84$ & $<-12.23$ & $-13.22 \pm 0.30$ & $\ldots$ \\
HIP 77900 B & $2014-04-12$ 08:38UT & $<-11.90$ & $<-11.80$ & $<-12.15$ & $<-12.22$ & $-13.04 \pm 0.27$ & $\ldots$ \\
HIP 78530 B & $2014-06-09$ 04:08UT & $<-11.20$ & $<-11.11$ & $<-11.38$ & $<-11.34$ & $-13.01 \pm 0.41$ \\
HIP 78530 B & $2014-06-09$ 05:14UT & $<-11.52$ & $<-11.25$ & $<-11.61$ & $<-11.42$ & $-13.06 \pm 0.36$ & $\ldots$ \\
\hline USco 1607-2242 & all epochs & $<-12.32$ & $<-11.99$ & $<-12.62$ & $<-12.85$ & $<-13.57$ & $\ldots$ \\
USco 1608-2232 & all epochs & $<-12.56$ & $<-11.45$ & $<-13.08$ & $<-12.85$ & $<-13.45$ & $\ldots$ \\
USco 1606-2335 & $2014-06-25$ & $<-11.86$ & $<-11.83$ & $-12.39 \pm 1.51$ & $-12.52 \pm 0.80$ & $-12.88 \pm 0.33$ \\
USco 1606-2335 & $2014-06-30$ & $<-12.09$ & $<-11.43$ & $-12.20 \pm 1.18$ & $-12.64 \pm 0.61$ & $-12.73 \pm 0.26$ & $-11.25 \pm 0.42$ \\
USco 1606-2335 & $2014-07-04<-12.21$ & $<-12.00$ & $-12.16 \pm 0.57$ & $-12.25 \pm 0.33$ & $-12.34 \pm 0.20$ & $-11.57 \pm 0.40$ & \\
USco 1606-2335 & $2014-07-29$ & $<-12.15$ & $<-12.00$ & $-12.30 \pm 0.62$ & $-12.54 \pm 0.37$ & $-12.65 \pm 0.23$ & $-11.87 \pm 0.37$ \\
USco 1610-2239 & $2014-06-30$ & $<-12.29$ & $<-12.20$ & $<-12.47$ & $<-12.89$ & $-13.46 \pm 0.27$ & $\ldots$ \\
USco 1610-2239 & $2014-07-01$ 03:26UT & $<-12.08$ & $<-11.20$ & $<-12.36$ & $<-12.70$ & $-13.27 \pm 0.33$ & $\ldots$ \\
USco 1610-2239 & $2014-07-0104: 04 U T$ & $<-12.46$ & $<-12.15$ & $<-12.54$ & $<-12.93$ & $-13.30 \pm 0.25$ & $\ldots$ \\
USco 1608-2315 & $2014-06-15$ & $<-12.10$ & $<-11.57$ & $-11.59 \pm 0.43$ & $-11.63 \pm 0.31$ & $-11.50 \pm 0.25$ & $-10.76 \pm 0.45$ \\
USco 1608-2315 & $2014-06-20$ & $<-12.10$ & $<-11.64$ & $-11.71 \pm 0.40$ & $-11.90 \pm 0.30$ & $-12.68 \pm 0.26$ & $-10.86 \pm 0.45$ \\
USco 1607-2211 & $2014-04-22$ & $<-12.08$ & $<-12.04$ & $<-12.37$ & $<-12.64$ & $-12.91 \pm 0.29$ & $-11.34 \pm 0.41$ \\
USco 1607-2211 & $2014-06-14$ & $<-12.28$ & $<-12.18$ & $<-12.44$ & $<-12.64$ & $\ldots$ & $\ldots$ \\
\hline
\end{tabular}

Notes. The accretion rate for each line is derived from Alcalá et al. (2017) and the one from the $10 \% \mathrm{H}_{\alpha}$ is derived from Natta et al. (2004). 